ESAIM: M2AN 54 (2020) 25-58

https://doi.org/10.1051/m2an/2019017
ESAIM: Mathematical Modelling and Numerical Analysis

www.esaim-m2an.org

\title{
PROJECTOR AUGMENTED-WAVE METHOD: AN ANALYSIS IN A ONE-DIMENSIONAL SETTING
}

\author{
Mi-SONG DUPUY*
}

\begin{abstract}
In this article, a numerical analysis of the projector augmented-wave (PAW) method is presented, restricted to the case of dimension one with Dirac potentials modeling the nuclei in a periodic setting. The PAW method is widely used in electronic ab initio calculations, in conjunction with pseudopotentials. It consists in replacing the original electronic Hamiltonian $H$ by a pseudoHamiltonian $H^{\mathrm{PAW}}$ via the PAW transformation acting in balls around each nuclei. Formally, the new eigenvalue problem has the same eigenvalues as $H$ and smoother eigenfunctions. In practice, the pseudoHamiltonian $H^{\mathrm{PAW}}$ has to be truncated, introducing an error that is rarely analyzed. In this paper, error estimates on the lowest PAW eigenvalue are proved for the one-dimensional periodic Schrödinger operator with double Dirac potentials.
\end{abstract}

Mathematics Subject Classification. 65N15, 65G99, 35P15.

Received December 13, 2017. Accepted February 18, 2019.

\section{INTRODUCTION}

In solid-state physics, to take advantage of the periodicity of the system, plane-wave methods are often the method of choice. However, Coulomb potentials located at each nucleus give rise to cusps on the eigenfunctions that impede the convergence rate of plane-wave expansions. Moreover, orthogonality to the core states implies fast oscillations of the valence state eigenfunctions that are difficult to approximate with plane-wave basis of moderate size. The PAW method [5] addresses both issues and has become a very popular tool over the years. It has been successfully implemented in different electronic structure simulation codes (ABINIT [13], VASP [11]) and has been adapted to the computations of various chemical properties [2,12]. It relies on an invertible transformation acting locally around each nucleus, mapping solutions of an atomic wave function to a smoother and slowly varying function. Moreover, because of the particular form of the PAW transformation, it is possible to use pseudopotentials $[10,14]$ in a consistent way. Hence, the PAW eigenfunctions are smoother and because of the invertibility of the PAW transformation, the sought eigenvalues are the same. However, the theoretical PAW equations involve infinite expansions which have to be truncated in practice. Doing so, the PAW method introduces an error that is rarely analyzed.

In this paper, the PAW method is applied to the one-dimensional double Dirac potential Hamiltonian whose eigenfunctions display a cusp at the location of the Dirac potentials that is reminiscent of the Kato cusp

Keywords and phrases. Eigenvalue problem, error analysis, electronic structure calculations, projector augmented-wave method.

Univ. Paris Diderot, Sorbonne Paris Cité, Laboratoire Jacques-Louis Lions, UMR 7598, UPMC, CNRS, 75205 Paris, France.

*Corresponding author: dupuy@math.univ-paris-diderot.fr 
condition [9]. Error estimates on the lowest PAW eigenvalue are proved for several choices of PAW parameters. The present analysis relies on some results on the variational PAW method (VPAW method) [3, 4] which is a slight modification of the original PAW method. Contrary to the PAW method, the VPAW generalized eigenvalue problem is in one-to-one correspondence with the original eigenvalue problem. By estimating the difference between the PAW and VPAW generalized eigenvalue problems, error estimates on the lowest PAW generalized eigenvalue are found.

\section{The PAW Method in A OnE-Dimensional Setting}

A general overview of the VPAW and PAW methods for 3-D electronic Hamiltonians may be found in [4] for the molecular setting and in [3] for crystals. Here, the presentation of the VPAW and PAW methods is limited to the application to the 1-D periodic Schrödinger operator with double Dirac potentials.

\subsection{The double Dirac potential Schrödinger operator}

We are interested in the lowest eigenvalue of the 1-D periodic Schrödinger operator $H$ on $L_{\text {per }}^{2}(0,1):=\{f \in$ $L_{\text {loc }}^{2}(\mathbb{R}) \mid f$ 1-periodic $\}$ with form domain $H_{\text {per }}^{1}(0,1):=\left\{f \in H_{\text {loc }}^{1}(\mathbb{R}) \mid f 1\right.$-periodic $\}$ :

$$
H=-\frac{\mathrm{d}^{2}}{\mathrm{~d} x^{2}}-Z_{0} \sum_{k \in \mathbb{Z}} \delta_{k}-Z_{a} \sum_{k \in \mathbb{Z}} \delta_{k+a}
$$

where $0<a<1, Z_{0}, Z_{a}>0$.

A mathematical analysis of this model has been carried out in [6]. The spectrum of $H$ is purely discrete. There are two negative eigenvalues $E_{0}=-\omega_{0}^{2}$ and $E_{1}=-\omega_{1}^{2}$ which are given by the zeros of the function

$$
f(\omega)=2 \omega^{2}(1-\cos h(\omega))+\left(Z_{0}+Z_{a}\right) \omega \sin h(\omega)-Z_{0} Z_{a} \sin h(a \omega) \sin h((1-a) \omega) .
$$

The corresponding eigenfunctions are

$$
\psi_{k}(x)= \begin{cases}A_{1, k} \cos h\left(\omega_{k} x\right)+B_{1, k} \sin h\left(\omega_{k} x\right), & 0 \leq x \leq a, \\ A_{2, k} \cos h\left(\omega_{k} x\right)+B_{2, k} \sin h\left(\omega_{k} x\right), & a \leq x \leq 1,\end{cases}
$$

where the coefficients $A_{1, k}, A_{2, k}, B_{1, k}$ and $B_{2, k}$ are determined by the continuity conditions and the derivative jumps at 0 and $a$.

There is an infinity of positive eigenvalues $E_{k+2}=\omega_{k+2}^{2}$ which are given by the $k$ th zero of the function:

$$
f(\omega)=2 \omega^{2}(1-\cos (\omega))+\left(Z_{0}+Z_{a}\right) \omega \sin (\omega)+Z_{0} Z_{a} \sin (a \omega) \sin ((1-a) \omega),
$$

and the corresponding eigenfunctions $H \psi_{k}=\omega_{k}^{2} \psi_{k}$ are

$$
\psi_{k}(x)= \begin{cases}A_{1, k} \cos \left(\omega_{k} x\right)+B_{1, k} \sin \left(\omega_{k} x\right), & 0 \leq x \leq a \\ A_{2, k} \cos \left(\omega_{k} x\right)+B_{2, k} \sin \left(\omega_{k} x\right), & a \leq x \leq 1,\end{cases}
$$

where again the coefficients $A_{1, k}, A_{2, k}, B_{1, k}$ and $B_{2, k}$ are determined by the continuity conditions and the derivative jumps at 0 and $a$. Notice that the eigenfunctions of $H$ have a first derivative jump that is similar to the Kato cusp condition satisfied by the solutions of 3D electronic Hamiltonian [9]:

$$
\psi_{k}^{\prime}\left(0_{+}\right)-\psi_{k}^{\prime}\left(0_{-}\right)=-Z_{0} \psi_{k}(0) .
$$

Because of these derivative jumps, Fourier methods to solve the eigenvalue problem

$$
\text { Find }\left(E_{k}, \psi_{k}\right) \in \mathbb{R} \times H_{\text {per }}^{1}(0,1) \text { such that } H \psi_{k}=E_{k} \psi_{k}
$$

slowly converge. The PAW method is one way to circumvent this difficulty. 


\subsection{The PAW method}

\subsubsection{General principle}

The PAW method consists in replacing the original eigenvalue problem $H \psi=E \psi$ by the generalized eigenvalue problem

$$
\left(\operatorname{Id}+T^{*}\right) H(\operatorname{Id}+T) \widetilde{\psi}=E\left(\operatorname{Id}+T^{*}\right)(\operatorname{Id}+T) \widetilde{\psi},
$$

where $\mathrm{Id}+T$ is an invertible operator. It is clear that $(2.4)$ is equivalent to $H \psi=E \psi$ where $\psi=(\operatorname{Id}+T) \widetilde{\psi}$.

The transformation $T$ is the sum of two operators acting in regions near the atomic sites that

$$
T=T_{0}+T_{a}, \quad T_{0}=\sum_{i=0}^{\infty}\left(\phi_{i}^{0}-\widetilde{\phi}_{i}^{0}\right)\left\langle\widetilde{p}_{i}^{0}, \cdot\right\rangle, \quad T_{a}=\sum_{i=0}^{\infty}\left(\phi_{i}^{a}-\widetilde{\phi}_{i}^{a}\right)\left\langle\widetilde{p}_{i}^{a}, \cdot\right\rangle,
$$

where for $f, g \in L_{\text {per }}^{2}(0,1),\langle f, g\rangle:=\int_{0}^{1} f(x) g(x) \mathrm{d} x$ denotes the $L_{\mathrm{per}}^{2}(0,1)$ scalar product. The operators $T_{0}$ and $T_{a}$ act respectively on $\bigcup_{k \in \mathbb{Z}}[-\eta+k, \eta+k]$ and $\bigcup_{k \in \mathbb{Z}}[a-\eta+k, a+\eta+k]$. To ensure the invertibility of the operator Id $+T$, these regions do not overlap. Thus $T_{0}$ and $T_{a}$ satisfy $T_{0} T_{a}=T_{a} T_{0}=0$ and the PAW cut-off radius $\eta$ must be chosen such that $\eta<a-\eta$ and $a+\eta<1-\eta$ hence $\eta<a / 2$.

The atomic wave functions $\left(\phi_{j}^{0}\right)_{j \in \mathbb{N}}$ are solutions of an atomic eigenvalue problem

$$
H_{0} \phi_{j}^{0}:=-\frac{\mathrm{d}^{2} \phi_{j}^{0}}{\mathrm{~d} x^{2}}-Z_{0} \sum_{k \in \mathbb{Z}} \delta_{k} \phi_{j}^{0}=\epsilon_{j}^{0} \phi_{j}^{0},
$$

and the pseudo wave functions $\left(\widetilde{\phi}_{j}^{0}\right)_{j \in \mathbb{N}}$ and the projector functions $\left(\widetilde{p}_{j}^{0}\right)_{j \in \mathbb{N}}$ are 1-periodic and satisfy the following conditions:

(1) for each $j \in \mathbb{N}$,

- for $x \in \mathbb{R} \backslash \bigcup_{k \in \mathbb{Z}}[-\eta+k, \eta+k], \widetilde{\phi}_{j}^{0}(x)=\phi_{j}^{0}(x)$;

- $\widetilde{\phi}_{j}^{0}$ restricted to $\bigcup_{k \in \mathbb{Z}}[-\eta+k, \eta+k]$ is a smooth function;

(2) for each $j \in \mathbb{N}$, supp $\widetilde{p}_{j}^{0} \subset \bigcup_{k \in \mathbb{Z}}[-\eta+k, \eta+k]$;

(3) the families $\left(\left.\widetilde{\phi}_{j}^{0}\right|_{[-\eta, \eta]}\right)_{j \in \mathbb{N}}$ and $\left(\left.\widetilde{p}_{j}^{0}\right|_{[-\eta, \eta]}\right)_{j \in \mathbb{N}}$ form a Riesz basis of $L^{2}(-\eta, \eta)$, i.e.

$$
\forall j, k \in \mathbb{N}, \int_{-\eta}^{\eta} \widetilde{p}_{k}^{0}(x) \widetilde{\phi}_{j}^{0}(x) \mathrm{d} x=\delta_{k j},
$$

and for any $f \in L^{2}(-\eta, \eta)$, we have

$$
\sum_{k=0}^{\infty}\left\langle\widetilde{p}_{k}^{0}, f\right\rangle \widetilde{\phi}_{k}^{0}(x)=f(x), \quad \text { for a.a. } x \in \bigcup_{k \in \mathbb{Z}}[-\eta+k, \eta+k] .
$$

This property is generally referred to as the completeness assumption in the original paper by Blöchl. This assumption is crucial in the PAW formulation since it allows to split physical quantities in a convenient way (see Eq. (2.6) below).

Similarly, $\left(\phi_{i}^{a}\right)_{i \in \mathbb{N}^{*}}$ are eigenfunctions of the operator $H_{a}=-\frac{\mathrm{d}^{2}}{\mathrm{~d} x^{2}}-Z_{0} \sum_{k \in \mathbb{Z}} \delta_{a+k}$, the pseudo wave functions $\left(\widetilde{\phi}_{j}^{a}\right)_{j \in \mathbb{N}^{*}}$ and the projector functions $\left(\widetilde{p}_{j}^{a}\right)_{j \in \mathbb{N}^{*}}$ are defined as above.

The completeness assumption (2.5) enables one to write the expression of $\left(\mathrm{Id}+T^{*}\right) H(\mathrm{Id}+T)$ and $\left(\operatorname{Id}+T^{*}\right)(\operatorname{Id}+T)$ as

$$
\left(\operatorname{Id}+T^{*}\right) H(\operatorname{Id}+T)=H+\sum_{\substack{i, j=0 \\ I=\{0, a\}}}^{\infty} \widetilde{p}_{i}^{I}\left(\left\langle\phi_{i}^{I}, H \phi_{j}^{I}\right\rangle-\left\langle\widetilde{\phi}_{i}^{I}, H \widetilde{\phi}_{j}^{I}\right\rangle\right)\left\langle\widetilde{p}_{j}^{I}, \cdot\right\rangle,
$$


and

$$
\left(\mathrm{Id}+T^{*}\right)(\operatorname{Id}+T)=\operatorname{Id}+\sum_{\substack{i, j=0 \\ I=\{0, a\}}}^{\infty} \widetilde{p}_{i}^{I}\left(\left\langle\phi_{i}^{I}, \phi_{j}^{I}\right\rangle-\left\langle\widetilde{\phi}_{i}^{I}, \widetilde{\phi}_{j}^{I}\right\rangle\right)\left\langle\widetilde{p}_{j}^{I}, \cdot\right\rangle
$$

Remark 2.1. It can be shown that $\mathrm{Id}+T$ is invertible provided that $\left(\phi_{i}-\widetilde{\phi}_{i}\right)_{i \in \mathbb{N}}$ is a family of linearly independent functions (see Prop. 1.3.1 in [1]). Since we focus on the error resulting of the truncation of equations (2.6) and (2.7) (and of Eq. (2.8) below for the version with pseudopotentials), we will not discuss this further.

\subsubsection{Introduction of a pseudopotential}

Further modifications of the PAW formulation are possible based on the introduction of the pseudopotentials. The pseudo wave functions $\widetilde{\phi}_{i}^{0}$ (resp. $\widetilde{\phi}_{i}^{a}$ ) are equal to $\phi_{i}^{0}$ (resp. $\phi_{i}^{a}$ ) outside $\bigcup_{k \in \mathbb{Z}}[-\eta+k, \eta+k]$ (resp. $\bigcup_{k \in \mathbb{Z}}[a-$ $\eta+k, a+\eta+k])$. Using the periodicity of these functions, the integrals appearing in (2.6) can be truncated to the interval $(-\eta, \eta)$ (resp. $(a-\eta, a+\eta))$. Doing so, another expression of $\left(\operatorname{Id}+T^{*}\right) H(\operatorname{Id}+T)$ can be obtained:

$$
\left(\mathrm{Id}+T^{*}\right) H(\mathrm{Id}+T)=H+\sum_{\substack{i, j=0 \\ I=\{0, a\}}}^{\infty} \tilde{p}_{i}^{I}\left(\left\langle\phi_{i}^{I}, H \phi_{j}^{I}\right\rangle_{I, \eta}-\left\langle\widetilde{\phi}_{i}^{I}, H \widetilde{\phi}_{j}^{I}\right\rangle_{I, \eta}\right)\left\langle\tilde{p}_{j}^{I}, \cdot \cdot\right\rangle,
$$

where

$$
\langle f, g\rangle_{I, \eta}= \begin{cases}\int_{-\eta}^{\eta} f(x) g(x) \mathrm{d} x, & \text { when } I=0, \\ \int_{a-\eta}^{a+\eta} f(x) g(x) \mathrm{d} x, & \text { when } I=a .\end{cases}
$$

Using this expression of the operator $H^{\mathrm{PAW}}$, it is possible to introduce a smooth 1-periodic potential $\chi_{\epsilon}=$ $\sum_{k \in \mathbb{Z}} \frac{1}{\epsilon} \chi\left(\frac{-k}{\epsilon}\right)$ with $\epsilon \leq \eta$, such that

(1) $\chi$ is an even, smooth and nonnegative function with support $[-1,1]$ and $\int_{-1}^{1} \chi(x) \mathrm{d} x=1$;

(2) $\chi_{\epsilon} \underset{\epsilon \rightarrow 0}{\longrightarrow} \sum_{k \in \mathbb{Z}} \delta_{k}$ in $H_{\text {per }}^{-1}(0,1)$.

The potential $\chi_{\epsilon}$ will be called a pseudopotential in the following.

Using the closure assumption (2.5) and that $\operatorname{supp} \chi_{\epsilon} \subset \bigcup_{k \in \mathbb{Z}}[-\eta+k, \eta+k]$, it is possible to show

$$
\left(\mathrm{Id}+T^{*}\right) H(\mathrm{Id}+T)=H_{\mathrm{ps}}+\sum_{\substack{i, j=0 \\ I=\{0, a\}}}^{\infty} \widetilde{p}_{i}^{I}\left(\left\langle\phi_{i}^{I}, H \phi_{j}^{I}\right\rangle_{I, \eta}-\left\langle\widetilde{\phi}_{i}^{I}, H_{\mathrm{ps}} \widetilde{\phi}_{j}^{I}\right\rangle_{I, \eta}\right)\left\langle\widetilde{p}_{j}^{I}, \cdot \cdot\right\rangle,
$$

with

$$
H_{\mathrm{ps}}=-\frac{\mathrm{d}^{2}}{\mathrm{~d} x^{2}}-Z_{0} \chi_{\epsilon}-Z_{a} \chi_{\epsilon}(\cdot-a)
$$

\subsection{The PAW method in practice}

For computational purposes, the double sums appearing in the operators (2.6)-(2.8) have to be truncated to some level $N$. Doing so, the identity $\psi=(\operatorname{Id}+T) \widetilde{\psi}$ is lost and the eigenvalues of the truncated equations are not equal to those of the original operator $H(2.1)$. The PAW method introduces an error that will be estimated in the rest of paper. First, we define the PAW functions appearing in (2.6)-(2.8).

\subsubsection{Generation of the PAW functions}

For the double Dirac potential Hamiltonian, the PAW functions are defined as follows. 
Atomic wave functions $\phi_{\boldsymbol{k}}^{\mathbf{0}}$. As mentioned earlier, the atomic wave functions $\left(\phi_{k}^{0}\right)_{1 \leq k \leq N}$ are eigenfunctions of the Hamiltonian $H_{0}$

$$
H_{0}=-\frac{\mathrm{d}^{2}}{\mathrm{~d} x^{2}}-Z_{0} \sum_{k \in \mathbb{Z}} \delta_{k}
$$

By parity, each eigenfunction of this operator is either even or odd. The odd eigenfunctions are $x \mapsto \sin (2 \pi k x)$ and the even ones are the 1-periodic functions such that

$$
\begin{cases}\phi_{0}^{0}(x):=\cos h\left(\omega_{0}\left(x-\frac{1}{2}\right)\right) & \text { for } x \in[0,1], \\ \phi_{k}^{0}(x):=\cos \left(\omega_{k}\left(x-\frac{1}{2}\right)\right) & \text { for } x \in[0,1], k \in \mathbb{N}^{*} .\end{cases}
$$

Since we want to solve difficulties due to the cusps, it seems reasonable to only keep the non-smooth eigenfunctions in the PAW formulation. In the sequel (and in particular in (2.10) and (2.13) below), only the non-smooth thus even eigenfunctions $\left(\phi_{i}^{0}\right)_{1 \leq i \leq N}$ are selected. The corresponding eigenvalues are denoted by $\left(\epsilon_{i}^{0}\right)_{1 \leq i \leq N}$ :

$$
H_{0} \phi_{i}^{0}=\epsilon_{i}^{0} \phi_{i}^{0} .
$$

Remark 2.2. An analysis of the PAW method with odd PAW functions is also provided. incorporating odd eigenfunctions in the PAW method with pseudopotentials can improve drastically the error on the PAW eigenvalue (see Thm. 3.5 below). The construction of the PAW method with odd functions is explained in Section 4.2.3.

Pseudo wave function $\widetilde{\phi}_{\boldsymbol{i}}^{0}$. The pseudo wave functions $\left(\widetilde{\phi}_{i}^{0}\right)_{1 \leq i \leq N} \in\left(H_{\mathrm{per}}^{1}(0,1)\right)^{N}$ are defined as follows:

(1) for $x \notin \bigcup_{k \in \mathbb{Z}}[-\eta+k, \eta+k], \widetilde{\phi}_{i}^{0}(x)=\phi_{i}^{0}(x)$.

(2) for $x \in \bigcup_{k \in \mathbb{Z}}[-\eta+k, \eta+k], \widetilde{\phi}_{i}^{0}$ is an even polynomial of degree at most $2 d-2, d \geq N$.

(3) $\widetilde{\phi}_{i}^{0}$ is $C^{d-1}$ at $\eta$ i.e. $\left(\widetilde{\phi}_{i}^{0}\right)^{(k)}(\eta)=\left(\phi_{i}^{0}\right)^{(k)}(\eta)$ for $0 \leq k \leq d-1$.

Projector functions $\widetilde{\boldsymbol{p}}_{\boldsymbol{i}}^{\mathbf{0}}$. Let $\rho$ be an even, positive, smooth function with support included in $[-1,1]$ and $\rho_{\eta}(t)=\sum_{k \in \mathbb{Z}} \rho\left(\frac{t-k}{\eta}\right)$. The projector functions $\left(\widetilde{p}_{i}^{0}\right)_{1 \leq i \leq N}$ are obtained by an orthogonalization procedure from the functions $p_{i}^{0}(t)=\rho_{\eta}(t) \widetilde{\phi}_{i}^{0}(t)$ in order to satisfy the duality condition:

$$
\left\langle\widetilde{p}_{i}^{0}, \widetilde{\phi}_{j}^{0}\right\rangle=\delta_{i j}
$$

More precisely, the matrix $B_{i j}:=\left\langle p_{i}^{0}, \widetilde{\phi}_{j}^{0}\right\rangle$ is computed and inverted to obtain the projector functions

$$
\widetilde{p}_{k}^{0}=\sum_{j=1}^{N}\left(B^{-1}\right)_{k j} p_{j}^{0} .
$$

The matrix $B$ is the Gram matrix of the functions $\left(\widetilde{\phi}_{j}^{0}\right)_{1 \leq j \leq N}$ for the weight $\rho_{\eta}$. The orthogonalization is possible only if the family $\left(\widetilde{\phi}_{i}^{0}\right)_{1 \leq i \leq N}$ is linearly independent - thus necessarily $d \geq N$.

\subsubsection{The eigenvalue problems}

For the case without pseudopotentials, the PAW eigenvalue problem is

$$
\text { Find }\left(E^{\mathrm{PAW}}, f\right) \in \mathbb{R} \times f \in H_{\mathrm{per}}^{1}(0,1), H^{\mathrm{PAW}} f=E^{\mathrm{PAW}} S^{\mathrm{PAW}} f,
$$

where

$$
H^{\mathrm{PAW}}=H+\sum_{\substack{i, j=1 \\ I=\{0, a\}}}^{N} \widetilde{p}_{i}^{I}\left(\left\langle\phi_{i}^{I}, H \phi_{j}^{I}\right\rangle-\left\langle\widetilde{\phi}_{i}^{I}, H \widetilde{\phi}_{j}^{I}\right\rangle\right)\left\langle\widetilde{p}_{j}^{I}, \cdot\right\rangle,
$$


and

$$
S^{\mathrm{PAW}}=\mathrm{Id}+\sum_{\substack{i, j=1 \\ I=\{0, a\}}}^{N} \widetilde{p}_{i}^{I}\left(\left\langle\phi_{i}^{I}, \phi_{j}^{I}\right\rangle-\left\langle\widetilde{\phi}_{i}^{I}, \widetilde{\phi}_{j}^{I}\right\rangle\right)\left\langle\widetilde{p}_{j}^{I}, \cdot\right\rangle .
$$

The practical interest in solving the eigenvalue problem (2.9) is very limited since this version of the PAW method does not remove the singularity caused by the Dirac potentials. The next eigenvalue problem where the Dirac potentials are replaced by smoother potentials is closer to the implementation of the PAW method in practice.

For the case with pseudopotentials, the PAW eigenvalue problem becomes

$$
\text { Find }\left(E_{\mathrm{ps}}^{\mathrm{PAW}}, f\right) \in \mathbb{R} \times f \in H_{\mathrm{per}}^{1}(0,1), H_{\mathrm{ps}}^{\mathrm{PAW}} f=E_{\mathrm{ps}}^{\mathrm{PAW}} S^{\mathrm{PAW}} f,
$$

where

$$
H_{\mathrm{ps}}^{\mathrm{PAW}}=H_{\mathrm{ps}}+\sum_{\substack{i, j=1 \\ I=\{0, a\}}}^{N} \widetilde{p}_{i}^{I}\left(\left\langle\phi_{i}^{I}, H \phi_{j}^{I}\right\rangle-\left\langle\widetilde{\phi}_{i}^{I}, H_{\mathrm{ps}} \widetilde{\phi}_{j}^{I}\right\rangle\right)\left\langle\widetilde{p}_{j}^{I}, \cdot\right\rangle
$$

and

$$
S^{\mathrm{PAW}}=\mathrm{Id}+\sum_{\substack{i, j=1 \\ I=\{0, a\}}}^{N} \widetilde{p}_{i}^{I}\left(\left\langle\phi_{i}^{I}, \phi_{j}^{I}\right\rangle-\left\langle\widetilde{\phi}_{i}^{I}, \widetilde{\phi}_{j}^{I}\right\rangle\right)\left\langle\widetilde{p}_{j}^{I}, \cdot\right\rangle .
$$

If the projector functions $\left(\widetilde{p}_{i}\right)_{1 \leq i \leq N}$ are smooth, then the eigenfunctions $f$ in $(2.12)$ are smooth as well, and their plane-wave expansions converge very quickly. Suppose that the difference $\left|E_{\mathrm{ps}}^{\mathrm{PAW}}-E\right|$ is smaller than a desired accuracy. Since the plane-wave discretization of the original eigenvalue problem converges slowly, it will be computationally more interesting to solve (2.12). However, an estimate on the difference $\left|E_{\mathrm{ps}}^{\mathrm{PAW}}-E\right|$ is needed in order to justify the use of the PAW method. To the best of our knowledge, there exists no estimation of this error except a heuristic analysis in the seminal work of Blöchl ([5], Sects. VII.B and VII.C). However, his analysis relies on an expansion of the eigenvalue in $f-\sum_{i=1}^{N}\left\langle\widetilde{p}_{i}, f\right\rangle \widetilde{\phi}_{i}$ which goes to 0 if the families $\left(\widetilde{p}_{i}\right)_{i \in \mathbb{N}^{*}}$ and $\left(\widetilde{\phi}_{i}\right)_{i \in \mathbb{N}^{*}}$ form a Riesz basis, but a convergence rate of the expansion in the Riesz basis is not given. Moreover the inclusion of a pseudopotential in the PAW treatment is not taken into account.

The goal of this paper is to provide error estimates on the lowest PAW eigenvalue of problems (2.9) and (2.12). To prove this result, the PAW method is interpreted as a perturbation of the VPAW method introduced in $[3,4]$ which has the same eigenvalues as the original problem. In the following, when we refer to the PAW method, it will be to the truncated equations (2.9) or (2.12).

\subsection{The VPAW method}

The analysis of the PAW method relies on the connection between the VPAW and the PAW methods. A brief description of the VPAW method is given in this subsection.

Like the PAW method, the principle of the VPAW method consists in replacing the original eigenvalue problem

$$
H \psi=E \psi,
$$

by the generalized eigenvalue problem:

$$
\left(\operatorname{Id}+T_{N}^{*}\right) H\left(\operatorname{Id}+T_{N}\right) \widetilde{\psi}=E\left(\operatorname{Id}+T_{N}\right)\left(\operatorname{Id}+T_{N}\right) \widetilde{\psi},
$$

where $\mathrm{Id}+T_{N}$ is an invertible operator. Thus both problems have the same eigenvalues and it is straightforward to recover the eigenfunctions of the former from the generalized eigenfunctions of the latter:

$$
\psi=\left(\mathrm{Id}+T_{N}\right) \widetilde{\psi}
$$


$T_{N}$ is also the sum of two operators acting near the atomic sites

$$
T_{N}=T_{0, N}+T_{a, N}
$$

To define $T_{0, N}$, we fix an integer $N$ and a radius $0<\eta<\min \left(\frac{a}{2}, \frac{1-a}{2}\right)$ so that $T_{0, N}$ and $T_{a, N}$ act on two disjoint regions $\bigcup_{k \in \mathbb{Z}}[-\eta+k, \eta+k]$ and $\bigcup_{k \in \mathbb{Z}}[a-\eta+k, a+\eta+k]$ respectively.

The operators $T_{0, N}$ and $T_{a, N}$ are given by

$$
T_{0, N}=\sum_{i=1}^{N}\left(\phi_{i}^{0}-\widetilde{\phi}_{i}^{0}\right)\left\langle\widetilde{p}_{i}^{0}, \cdot\right\rangle, \quad T_{a, N}=\sum_{i=1}^{N}\left(\phi_{i}^{a}-\widetilde{\phi}_{i}^{a}\right)\left\langle\widetilde{p}_{i}^{a}, \cdot\right\rangle,
$$

with the same functions $\phi_{i}^{I}, \widetilde{\phi}_{i}^{I}$ and $\widetilde{p}_{i}^{I}, I=0, a$ as in Section 2.2. The only difference with the PAW method is that the sums appearing in (2.17) are finite, thereby avoiding a truncation error.

In the following, the VPAW operators are denoted by

$$
H^{\mathrm{VPAW}}=\left(\mathrm{Id}+T_{N}^{*}\right) H\left(\mathrm{Id}+T_{N}\right),
$$

and

$$
S^{\mathrm{VPAW}}=\left(\mathrm{Id}+T_{N}^{*}\right)\left(\mathrm{Id}+T_{N}\right),
$$

A full analysis of the VPAW method can be found in [3]. In this paper, it has been proved that the cusps at 0 and $a$ of the eigenfunctions $\widetilde{\psi}$ are reduced by a factor $\eta^{2 N}$ but the $d$ th derivative jumps introduced by the pseudo wave functions $\widetilde{\phi}_{k}$ blow up as $\eta$ goes to 0 at the rate $\eta^{1-d}$. Using Fourier methods to solve (2.15), we observe an acceleration of convergence that can be tuned by the VPAW parameters $\eta$ (the cut-off radius), $N$ (the number of PAW functions used at each site) and $d$ (the smoothness of the PAW pseudo wave functions).

\section{MAIN RESULTS}

The PAW method is well-posed if the projector functions $\left(\widetilde{p}_{i}^{I}\right)_{1 \leq i \leq N}$ are well-defined. This question has already been addressed in [3] where it is shown that we simply need to take $\eta<\eta_{0}$ for some positive $\eta_{0}$.

Assumption 3.1. Let $\eta_{0}>0$ such that for all $0<\eta<\eta_{0}$, the projector functions $\left(\widetilde{p}_{i}\right)_{1 \leq i \leq N}$ in Section 2.3.1 are well-defined.

Moreover since the analysis of the PAW error requires the VPAW method to be well-posed, the matrix $\left(\left\langle\widetilde{p}_{j}^{I}, \phi_{k}^{I}\right\rangle\right)_{1 \leq j, k \leq N}$ is assumed to be invertible for $0<\eta \leq \eta_{0}$.

Assumption 3.2. For all $0<\eta<\eta_{0}$, the matrix $\left(\left\langle\widetilde{p}_{j}^{I}, \phi_{k}^{I}\right\rangle\right)_{1 \leq j, k \leq N}$ is invertible.

Under these assumptions, the following theorems are established. Proofs are gathered in Section 4.

\subsection{PAW method without pseudopotentials}

Theorem 3.3. Let $\phi_{i}^{I}, \widetilde{\phi}_{i}^{I}$ and $\widetilde{p}_{i}^{I}$, for $i=1, \ldots, N$ and $I \in\{0, a\}$ be the functions defined in Section 2.3.1. Let $E^{\mathrm{PAW}}$ be the lowest eigenvalue of the generalized eigenvalue problem (2.9). Let $E_{0}$ be the lowest eigenvalue of $H$ (2.1). Then under Assumptions 3.1 and 3.2, there exists a positive constant $C$ independent of $\eta$ such that for all $0<\eta \leq \eta_{0}$

$$
-C \eta \leq E^{\mathrm{PAW}}-E_{0} \leq C \eta^{2 N} .
$$

The constant $C$ appearing in (3.1) (and in the theorems that will follow) depends on the other PAW parameters $N$ and $d$ in a nontrivial way. The upper bound is proved by using the VPAW eigenfunction $\widetilde{\psi}$ associated to the lowest eigenvalue $E_{0}$ for which we have precise estimates of the difference between the operators $H_{\mathrm{ps}}^{\mathrm{PAW}}$ and $H^{\mathrm{VPAW}}$. As expected (and confirmed by numerical simulations in Sect. 5.1.1) the PAW method without pseudopotentials is not variational. Moreover as the Dirac delta potentials are not removed, Fourier methods applied to the eigenvalue problem (2.9) converge slowly. 


\subsection{PAW method with pseudopotentials}

The following theorems are stated for $\epsilon=\eta$, i.e. when the support of the pseudopotential is equal to the acting region of the PAW method. Indeed, in the proof of Theorem 3.4, it appears that poorer estimates are obtained when a pseudopotential $\chi_{\epsilon}$ with $\epsilon<\eta$ is used.

Theorem 3.4. Let $\phi_{i}^{I}, \widetilde{\phi}_{i}^{I}$ and $\widetilde{p}_{i}^{I}$, for $i=1, \ldots, N$ and $I \in\{0, a\}$ be the functions defined in Section 2.3.1. Let $E_{\mathrm{ps}}^{\mathrm{PAW}}$ be the lowest eigenvalue of the generalized eigenvalue problem (2.12). Let $E_{0}$ be the lowest eigenvalue of $H$ (2.1). Then under Assumptions 3.1 and 3.2, there exists a positive constant $C$ independent of $\eta$ such that for all $0<\eta \leq \eta_{0}$

$$
-C \eta \leq E_{\mathrm{ps}}^{\mathrm{PAW}}-E_{0} \leq C \eta^{2} .
$$

As the PAW cut-off radius $\eta$ goes to 0 , the lowest eigenvalue of the truncated PAW equations is closer to the exact eigenvalue. This is also observed in different implementations of the PAW method and is in fact one of the main guidelines: a small cutoff radius yields more accurate results $[7,8]$.

Introducing a pseudopotential in $H^{\mathrm{PAW}}$ worsens the upper bound on the PAW eigenvalue. This is due to our construction of the PAW method in Section 2.2 where only even PAW functions are considered. Incorporating odd PAW functions in the PAW treatment, it is possible to improve the upper bound on the PAW eigenvalue and recover the bound in Theorem 3.3 (see Sect. 4.2.3 for the construction of the odd PAW functions).

Theorem 3.5. Let $\phi_{i}^{I}, \widetilde{\phi}_{i}^{I}$ and $\widetilde{p}_{i}^{I}$, for $i=1, \ldots, N$ and $I \in\{0, a\}$ be the functions defined in Section 2.3.1. Let $\left(\widetilde{\theta}_{k}^{I}\right)_{1 \leq k \leq N}$ be the functions given by (4.28) and $\left(\widetilde{q}_{k}^{I}\right)_{1 \leq k \leq N}$ be the functions given by (4.30). Let $E_{\mathrm{ps}}^{\mathrm{PAW}}$ be the lowest eigenvalue of the generalized eigenvalue problem $H_{\mathrm{ps}}^{\mathrm{PAW}} f=E_{\mathrm{ps}}^{\mathrm{PAW}} S^{\mathrm{PAW}} f$ with $H_{\mathrm{ps}}^{\mathrm{PAW}}$ defined in (4.31). Let $E_{0}$ be the lowest eigenvalue of $H$ defined by (2.1). Then under Assumptions 3.1 and 3.2, there exists a positive constant $C$ independent of $\eta$ such that for all $0<\eta \leq \eta_{0}$

$$
-C \eta \leq E_{\mathrm{ps}}^{\mathrm{PAW}}-E_{0} \leq C \eta^{2 N} \text {. }
$$

\subsection{Error estimates of the plane-wave discretization of the PAW method with pseudopotentials}

The potential appearing in the PAW eigenvalue problem (2.12) is smooth. Hence eigenfunctions are smooth and convergence is faster than $\frac{1}{M^{n}}$ for any $n \in \mathbb{N}$. There is however a prefactor which depends on the $n-1$ th derivative of the eigenfunction. An estimate of the $L^{\infty}$-norm of the PAW eigenfunction is given by Lemma 4.7. Using this lemma, an error estimate of the plane-wave discretization of the eigenvalue problem (2.12) can be proved.

Theorem 3.6. Let $\phi_{i}^{I}, \widetilde{\phi}_{i}^{I}$ and $\widetilde{p}_{i}^{I}$, for $i=1, \ldots, N$ and $I \in\{0, a\}$ be the functions defined in Section 2.3.1. Let $E_{M}^{\mathrm{PAW}}$ be the lowest eigenvalue of the variational approximation of $(2.12)$, with $H_{\mathrm{ps}}^{\mathrm{PAW}}$ given by $(2.13)$ in a basis of $M$ plane waves. Let $E_{0}$ be the lowest eigenvalue of $H$ (2.1). Under Assumptions 3.1 and 3.2, there exists a positive constant $C$ independent of $\eta$ and $M$ such that for all $0<\eta<\eta_{0}$ and for any $n \in \mathbb{N}^{*}$

$$
\left|E_{M}^{\mathrm{PAW}}-E_{0}\right| \leq C\left(\eta+\frac{\eta^{2}}{(\eta M)^{n}}\right) .
$$

According to Theorem 3.6, if we want to compute $E_{0}$ up to a desired accuracy $\varepsilon$, then it suffices to choose the PAW cut-off radius $\eta$ equal to $\frac{\varepsilon}{C}$ and solve the PAW eigenvalue problem (2.12) with $M \geq \frac{1}{\eta}$ plane-waves.

Remark 3.7. Using more PAW functions does not improve the bound on the computed eigenvalue. It is due to the poor lower bound in Theorems 3.4 and 3.5. Should the PAW method with odd functions (Sect. 4.2.3) be variational, we would know a priori that $E_{\mathrm{ps}}^{\mathrm{PAW}} \geq E_{0}$. Therefore, we could prove the estimate

$$
0<E_{M}^{\mathrm{PAW}}-E_{0} \leq C\left(\eta^{2 N}+\frac{\eta^{2}}{(\eta M)^{n}}\right) .
$$


Hence taking a plane wave cut-off $M \geq \frac{1}{\eta}$ would ensure that the eigenvalue $E_{0}$ is computed up to an error of order $\mathcal{O}\left(\eta^{2 N}\right)$.

\section{Proofs of ERror estimates}

We introduce some notation used in the below proofs. Let $I \in\{0, a\}$ and

$$
\begin{aligned}
p^{I}(t) & :=\left(p_{1}^{I}(t), \ldots, p_{N}^{I}(t)\right)^{T} \in \mathbb{R}^{N}, \\
\widetilde{p}^{I}(t) & :=\left(\widetilde{p}_{1}^{I}(t), \ldots, \widetilde{p}_{N}^{I}(t)\right)^{T} \in \mathbb{R}^{N}, \\
\left\langle\widetilde{p}^{I}, f\right\rangle & :=\left(\left\langle\widetilde{p}_{1}^{I}, f\right\rangle, \ldots,\left\langle\widetilde{p}_{N}^{I}, f\right\rangle\right)^{T} \in \mathbb{R}^{N}, \forall f \in L_{\mathrm{per}}^{2}(0,1), \\
\Phi_{I}(t) & :=\left(\phi_{1}^{I}(t), \ldots, \phi_{N}^{I}(t)\right)^{T} \in \mathbb{R}^{N}, \\
\widetilde{\Phi}_{I}(t) & :=\left(\widetilde{\phi}_{1}^{I}(t), \ldots, \widetilde{\phi}_{N}^{I}(t)\right)^{T} \in \mathbb{R}^{N}, \\
A_{I} & :=\left(\left\langle p_{i}^{I}, \phi_{j}^{I}\right\rangle\right)_{1 \leq i, j \leq N} \in \mathbb{R}^{N \times N} .
\end{aligned}
$$

For $p \in[1, \infty]$, we denote by

$$
\|f\|_{p, \eta, I}=\left\{\begin{array}{ll}
\|f\|_{L^{p}(-\eta, \eta)}, & \text { if } I=0 \\
\|f\|_{L^{p}(a-\eta, a+\eta)}, & \text { if } I=a
\end{array} .\right.
$$

In the remainder of the paper, $C$ denotes a generic positive constant that does not depend on the PAW cut-off radius $\eta$.

\subsection{PAW method without pseudopotentials}

The main idea of the proof is to use that the PAW operator $H^{\mathrm{PAW}}(2.10)$ (respectively $S^{\mathrm{PAW}}(2.11)$ ) is close to the VPAW operator $H^{\mathrm{VPAW}}(2.18)$ (resp. $S^{\mathrm{VPAW}}(2.19)$ ), in a sense that will be clearly stated. Then it is possible to use this connection and bound the error on the PAW eigenvalue $E^{\mathrm{PAW}}$, since the VPAW generalized eigenvalue problem (2.15) has the same eigenvalues as the original eigenvalue problem (2.3).

Proposition 4.1. Let $H^{\mathrm{PAW}}, S^{\mathrm{PAW}}, H^{\mathrm{VPAW}}$ and $S^{\mathrm{VPAW}}$ be defined by equations (2.10), (2.11), (2.18) and (2.19) respectively. Then for $f \in H_{\mathrm{per}}^{1}(0,1)$, we have

$$
\left\langle f, H^{\mathrm{VPAW}} f\right\rangle=\left\langle f, H^{\mathrm{PAW}} f\right\rangle+2 \sum_{I=\{0, a\}}\left\langle\left\langle\widetilde{p}^{I}, f\right\rangle^{T}\left(\Phi_{I}-\widetilde{\Phi}_{I}\right), H\left(f-\left\langle\widetilde{p}^{I}, f\right\rangle^{T} \widetilde{\Phi}_{I}\right)\right\rangle,
$$

and

$$
\left\langle f, S^{\mathrm{VPAW}} f\right\rangle=\left\langle f, S^{\mathrm{PAW}} f\right\rangle+2 \sum_{I=\{0, a\}}\left\langle\left\langle\widetilde{p}^{I}, f\right\rangle^{T}\left(\Phi_{I}-\widetilde{\Phi}_{I}\right), f-\left\langle\widetilde{p}^{I}, f\right\rangle^{T} \widetilde{\Phi}_{I}\right\rangle .
$$

Proof. Let $f \in H_{\mathrm{per}}^{1}(0,1)$. By expanding $H^{\mathrm{VPAW}}=\left(\mathrm{Id}+T_{N}\right)^{*} H\left(\mathrm{Id}+T_{N}\right)$ and using that $T_{0, N}$ and $T_{a, N}$ act on strictly distinct region, we have

$$
\begin{aligned}
\left\langle f, H^{\mathrm{VPAW}} f\right\rangle= & \left\langle f+\sum_{I=\{0, a\}}\left\langle\widetilde{p}^{I}, f\right\rangle^{T}\left(\Phi_{I}-\widetilde{\Phi}_{I}\right), H\left(f+\sum_{I=\{0, a\}}\left\langle\widetilde{p}^{I}, f\right\rangle^{T}\left(\Phi_{I}-\widetilde{\Phi}_{I}\right)\right)\right\rangle \\
= & \langle f, H f\rangle+\sum_{I=\{0, a\}} 2\left\langle f, H\left\langle\widetilde{p}^{I}, f\right\rangle^{T}\left(\Phi_{I}-\widetilde{\Phi}_{I}\right)\right\rangle \\
& +\sum_{I=\{0, a\}}\left\langle\left\langle\widetilde{p}^{I}, f\right\rangle^{T}\left(\Phi_{I}-\widetilde{\Phi}_{I}\right), H\left\langle\widetilde{p}^{I}, f\right\rangle^{T}\left(\Phi_{I}-\widetilde{\Phi}_{I}\right)\right\rangle
\end{aligned}
$$




$$
\begin{aligned}
= & \langle f, H f\rangle+\sum_{I=\{0, a\}} 2\left\langle f, H\left\langle\widetilde{p}^{I}, f\right\rangle^{T}\left(\Phi_{I}-\widetilde{\Phi}_{I}\right)\right\rangle+\left\langle\left\langle\widetilde{p}^{I}, f\right\rangle^{T} \Phi_{I}, H\left\langle\widetilde{p}^{I}, f\right\rangle^{T} \Phi_{I}\right\rangle \\
& -2\left\langle\left\langle\widetilde{p}^{I}, f\right\rangle^{T} \widetilde{\Phi}_{I}, H\left\langle\widetilde{p}^{I}, f\right\rangle^{T} \Phi_{I}\right\rangle+\left\langle\left\langle\widetilde{p}^{I}, f\right\rangle^{T} \widetilde{\Phi}_{I}, H\left\langle\widetilde{p}^{I}, f\right\rangle^{T} \widetilde{\Phi}_{I}\right\rangle .
\end{aligned}
$$

Notice that for each $I$, we have

$$
\begin{aligned}
\left\langle\left\langle\widetilde{p}^{I}, f\right\rangle^{T} \widetilde{\Phi}_{I}, H\left\langle\widetilde{p}^{I}, f\right\rangle^{T} \Phi_{I}\right\rangle= & \left\langle\left\langle\widetilde{p}^{I}, f\right\rangle^{T} \widetilde{\Phi}_{I}, H\left\langle\widetilde{p}^{I}, f\right\rangle^{T}\left(\Phi_{I}-\widetilde{\Phi}_{I}\right)\right\rangle \\
& +\left\langle\left\langle\widetilde{p}^{I}, f\right\rangle^{T} \widetilde{\Phi}_{I}, H\left\langle\widetilde{p}^{I}, f\right\rangle^{T} \widetilde{\Phi}_{I}\right\rangle .
\end{aligned}
$$

Hence

$$
\begin{aligned}
\left\langle f, H^{\mathrm{VPAW}} f\right\rangle= & \langle f, H f\rangle+\sum_{I=\{0, a\}}\left\langle\left\langle\widetilde{p}^{I}, f\right\rangle^{T} \Phi_{I}, H\left\langle\widetilde{p}^{I}, f\right\rangle^{T} \Phi_{I}\right\rangle-\left\langle\left\langle\widetilde{p}^{I}, f\right\rangle^{T} \widetilde{\Phi}_{I}, H\left\langle\widetilde{p}^{I}, f\right\rangle^{T} \widetilde{\Phi}_{I}\right\rangle \\
& +2\left\langle f-\left\langle\widetilde{p}^{I}, f\right\rangle^{T} \widetilde{\Phi}_{I}, H\left\langle\widetilde{p}^{I}, f\right\rangle^{T}\left(\Phi_{I}-\widetilde{\Phi}_{I}\right)\right\rangle \\
= & \left\langle f, H^{\mathrm{PAW}} f\right\rangle+\sum_{I=\{0, a\}} 2\left\langle f-\left\langle\widetilde{p}^{I}, f\right\rangle^{T} \widetilde{\Phi}_{I}, H\left\langle\widetilde{p}^{I}, f\right\rangle^{T}\left(\Phi_{I}-\widetilde{\Phi}_{I}\right)\right\rangle .
\end{aligned}
$$

The second identity is proved the same way.

\subsubsection{Proof of the upper bound of Theorem 3.3}

The upper bound on the PAW eigenvalue is proved by replacing $f$ in Proposition 4.1 by the VPAW eigenfunction $\widetilde{\psi}$ and estimating the differences $\left\langle\widetilde{\psi}, H^{\mathrm{PAW}} \widetilde{\psi}\right\rangle-\left\langle\widetilde{\psi}, H^{\mathrm{VPAW}} \widetilde{\psi}\right\rangle$ and $\left\langle\widetilde{\psi}, S^{\mathrm{PAW}} \widetilde{\psi}\right\rangle-\left\langle\widetilde{\psi}, S^{\mathrm{VPAW}} \widetilde{\psi}\right\rangle$. By Lemma A.1 in the appendix, we only have a good bound on $\widetilde{\psi}_{e}-\left\langle\widetilde{p}^{I}, \widetilde{\psi}\right\rangle^{T} \widetilde{\Phi}_{I}$, where $\widetilde{\psi}_{e}$ is the even part of $\widetilde{\psi}$. This is however sufficient because of the parity of the PAW functions and of the operator $H$ close to the Dirac potentials. Before proving this upper bound, we will need the following result on the $H^{1}$-norm of the VPAW eigenfunction.

Lemma 4.2. Let $\widetilde{\psi}$ be an $L_{\mathrm{per}}^{2}$-normalized generalized eigenfunction associated to the lowest eigenvalue of the $V P A W$ eigenvalue problem defined in (2.15). Then there exists a positive constant $C$ independent of $\eta$ such that for all $0<\eta \leq \eta_{0}$

$$
\|\widetilde{\psi}\|_{H_{\mathrm{per}}^{1}} \leq C .
$$

Proof. The operator $H$ defined in (2.1) is coercive. A proof of this statement can be found in [6]. Let $\alpha>0$ be such that for all $f \in H_{\text {per }}^{1}(0,1)$

$$
\langle f, H f\rangle+\alpha\langle f, f\rangle \geq \frac{1}{2}\|f\|_{H_{\mathrm{per}}^{1}}^{2} .
$$

Then

$$
\left\langle\widetilde{\psi}, H^{\mathrm{VPAW}} \widetilde{\psi}\right\rangle+\alpha\left\langle\widetilde{\psi}, S^{\mathrm{VPAW}} \widetilde{\psi}\right\rangle \geq \frac{1}{2}\left\|\left(\mathrm{Id}+T_{N}\right) \widetilde{\psi}\right\|_{H_{\mathrm{per}}^{1}}^{2}
$$

By item 1 of Lemma A.3, we have

$$
\left\|T_{N} \widetilde{\psi}\right\|_{H_{\mathrm{per}}^{1}} \leq C \eta^{1 / 2}\|\widetilde{\psi}\|_{H_{\mathrm{per}}^{1}},
$$

for some positive constant $C$ independent of $\eta$. Hence, for $\eta$ sufficiently small, there exists a positive constant $C$ independent of $\eta$ such that

$$
\left(E_{0}+\alpha\right)\left\langle\widetilde{\psi}, S^{\mathrm{VPAW}} \widetilde{\psi}\right\rangle \geq C\|\widetilde{\psi}\|_{H_{\mathrm{per}}^{1}}^{2} .
$$


Using item 1 of Lemma B.1, we obtain

$$
C\|\widetilde{\psi}\|_{L_{\mathrm{per}}^{2}}^{2} \geq\|\widetilde{\psi}\|_{H_{\mathrm{per}}^{1}}^{2}
$$

and the result follows from the normalization of the eigenfunctions $\widetilde{\psi}$.

We now have all the necessary tools to prove the upper bound of Theorem 3.4.

Proof of the upper bound of Theorem 3.3. Let $\widetilde{\psi}$ be an $L_{\mathrm{per}}^{2}$-normalized eigenvector of the lowest eigenvalue of $H^{\mathrm{VPAW}} \widetilde{\psi}=E_{0} S^{\mathrm{VPAW}} \widetilde{\psi}$. Then by Proposition 4.1,

$$
\left\langle\widetilde{\psi}, H^{\mathrm{VPAW}} \widetilde{\psi}\right\rangle=\left\langle\widetilde{\psi}, H^{\mathrm{PAW}} \widetilde{\psi}\right\rangle+2 \sum_{I=\{0, a\}}\left\langle\widetilde{\psi}-\left\langle\widetilde{p}^{I}, \widetilde{\psi}\right\rangle^{T} \widetilde{\Phi}_{I},\left\langle\widetilde{p}^{I}, \widetilde{\psi}\right\rangle^{T} H\left(\Phi_{I}-\widetilde{\Phi}_{I}\right)\right\rangle .
$$

Equation (A.2) yields

$$
\widetilde{\psi}-\left\langle\widetilde{p}^{I}, \widetilde{\psi}\right\rangle^{T} \widetilde{\Phi}_{I}=\psi-\left\langle A_{I}^{-1} p^{I}, \psi\right\rangle^{T} \Phi_{I}
$$

Thus form Proposition 4.1 we have:

$$
\begin{aligned}
\left\langle\widetilde{\psi}, H^{\mathrm{VPAW}} \widetilde{\psi}\right\rangle & =\left\langle\widetilde{\psi}, H^{\mathrm{PAW}} \widetilde{\psi}\right\rangle+2 \sum_{I=\{0, a\}}\left\langle\psi-\left\langle A_{I}^{-1} p^{I}, \psi\right\rangle^{T} \Phi_{I}, H\left\langle\widetilde{p}^{I}, \widetilde{\psi}\right\rangle^{T}\left(\Phi_{I}-\widetilde{\Phi}_{I}\right)\right\rangle \\
& =\left\langle\widetilde{\psi}, H^{\mathrm{PAW}} \widetilde{\psi}\right\rangle+2 \sum_{I=\{0, a\}}\left\langle E_{0} \psi-\left\langle A_{I}^{-1} p^{I}, \psi\right\rangle^{T} \mathcal{E}^{I} \Phi_{I},\left\langle\widetilde{p}^{I}, \widetilde{\psi}\right\rangle^{T}\left(\Phi_{I}-\widetilde{\Phi}_{I}\right)\right\rangle,
\end{aligned}
$$

where we used $H \Phi_{I}=\mathcal{E}^{I} \Phi_{I}$ in $(I-\eta, I+\eta)$ for $I \in\{0, a\}$. By Lemma A.1,

$$
\left\|E_{0} \psi_{e}-\left\langle A_{I}^{-1} p^{I}, \psi\right\rangle^{T} \mathcal{E}^{I} \Phi_{I}\right\|_{\infty, \eta, I} \leq C \eta^{2 N-2}
$$

So for each $I$,

$$
\begin{aligned}
& \left|\left\langle E_{0} \psi_{e}-\left\langle A_{I}^{-1} p^{I}, \psi\right\rangle^{T} \mathcal{E}^{I} \Phi_{I},\left\langle\widetilde{p}^{I}, \widetilde{\psi}\right\rangle^{T}\left(\Phi_{I}-\widetilde{\Phi}_{I}\right)\right\rangle\right| \\
& \leq\left\|E_{0} \psi_{e}-\left\langle A_{I}^{-1} p^{I}, \psi\right\rangle^{T} \mathcal{E}^{I} \Phi_{I}\right\|_{\infty, \eta, I}\left\|\left\langle\widetilde{p}^{I}, \widetilde{\psi}\right\rangle^{T}\left(\Phi_{I}-\widetilde{\Phi}_{I}\right)\right\|_{1, \eta, I} .
\end{aligned}
$$

By item 1 of Lemma A.3, we have

$$
\left\|\left\langle\widetilde{p}^{I}, \widetilde{\psi}\right\rangle^{T}\left(\Phi_{I}-\widetilde{\Phi}_{I}\right)\right\|_{1, \eta, I} \leq C \eta^{2}\|\widetilde{\psi}\|_{H_{\mathrm{per}}^{1}} \leq C \eta^{2}
$$

where we bound $\|\widetilde{\psi}\|_{H_{\mathrm{per}}^{1}}$ by means of Lemma 4.2. Hence, using Lemma A.1, we obtain

$$
\left|\left\langle E_{0} \psi-\left\langle A_{I}^{-1} p^{I}, \psi\right\rangle^{T} \mathcal{E}^{I} \Phi_{I},\left\langle\widetilde{p}^{I}, \widetilde{\psi}\right\rangle^{T}\left(\Phi_{I}-\widetilde{\Phi}_{I}\right)\right\rangle\right| \leq C \eta^{2 N} .
$$

Going back to equation (4.3),

$$
\begin{aligned}
E_{0}\left\langle\widetilde{\psi}, S^{\mathrm{VPAW}} \widetilde{\psi}\right\rangle+C \eta^{2 N} & \geq\left\langle\widetilde{\psi}, H^{\mathrm{PAW}} \widetilde{\psi}\right\rangle \\
& \geq E^{\mathrm{PAW}}\left\langle\widetilde{\psi}, S^{\mathrm{PAW}} \widetilde{\psi}\right\rangle .
\end{aligned}
$$


By item 4 of Lemma B.1 and Lemma 4.2, it holds

$$
\left|\left\langle\widetilde{\psi}, S^{\mathrm{VPAW}} \widetilde{\psi}\right\rangle-\left\langle\widetilde{\psi}, S^{\mathrm{PAW}} \widetilde{\psi}\right\rangle\right| \leq C \eta^{2 N+2}
$$

Hence, together with item 2 of Lemma B.1, we obtain

$$
\left\langle\widetilde{\psi}, S^{N} \widetilde{\psi}\right\rangle\left(E^{\mathrm{PAW}}-E_{0}\right) \leq C \eta^{2 N}
$$

The VPAW eigenfunction $\widetilde{\psi}$ is $L_{\text {per }}^{2}$-normalized, thus item 5 of Lemma B.1 completes the proof.

\subsubsection{Proof of the lower bound of Theorem 3.3}

The proof of the lower bound will roughly follow the same steps as the proof of the upper bound of Theorem 3.3 .

Lemma 4.3. Let $f$ be an $L_{\mathrm{per}}^{2}$-normalized generalized eigenfunction associated to the lowest generalized eigenvalue of (2.9). Then there exists a positive constant $C$ independent of $\eta$ such that for all $0<\eta \leq \eta_{0}$

$$
\|f\|_{H_{\mathrm{per}}^{1}} \leq C .
$$

Proof. We proceed as in the proof of Lemma 4.2. Let $\alpha$ be the coercivity constant of $H$ and $f$ be an $L_{\text {per- }}^{2}$ normalized eigenfunction associated to the lowest eigenvalue of (2.9). Then we have

$$
\alpha\langle f, f\rangle+\langle f, H f\rangle \geq \frac{1}{2}\|f\|_{H_{\mathrm{per}}^{1}}^{2} .
$$

From equation (2.10), we can prove that we have

$$
\left\langle f, H^{\mathrm{PAW}} f\right\rangle=\langle f, H f\rangle+\sum_{I \in\{0, a\}}\left\langle\left\langle\widetilde{p}^{I}, f\right\rangle^{T}\left(\Phi_{I}+\widetilde{\Phi}_{I}\right), H\left\langle\widetilde{p}^{I}, f\right\rangle^{T}\left(\Phi_{I}-\widetilde{\Phi}_{I}\right)\right\rangle .
$$

Hence, we have

$$
\begin{aligned}
& \alpha\langle f, f\rangle+\left\langle f, H^{\mathrm{PAW}} f\right\rangle-\sum_{I \in\{0, a\}}\left\langle\left\langle\widetilde{p}^{I}, f\right\rangle^{T}\left(\Phi_{I}+\widetilde{\Phi}_{I}\right), H\left\langle\widetilde{p}^{I}, f\right\rangle^{T}\left(\Phi_{I}-\widetilde{\Phi}_{I}\right)\right\rangle \geq \frac{1}{2}\|f\|_{H_{\mathrm{per}}^{1}}^{2} \\
& \alpha\langle f, f\rangle+\left\langle f, H^{\mathrm{PAW}} f\right\rangle \geq \frac{1}{2}\|f\|_{H_{\mathrm{per}}^{1}}^{2}-C \sum_{I \in\{0, a\}}\left\|\left\langle\widetilde{p}^{I}, f\right\rangle^{T}\left(\Phi_{I}+\widetilde{\Phi}_{I}\right)\right\|_{H^{1}, \eta, I}\left\|\left\langle\widetilde{p}^{I}, f\right\rangle^{T}\left(\Phi_{I}-\widetilde{\Phi}_{I}\right)\right\|_{H^{1}, \eta, I} .
\end{aligned}
$$

Combining items 1, 3 and 4 of Lemma A.3, we obtain

$$
\left\|\left\langle\widetilde{p}^{I}, f\right\rangle^{T}\left(\Phi_{I}+\widetilde{\Phi}_{I}\right)\right\|_{H^{1}, \eta, I}\left\|\left\langle\widetilde{p}^{I}, f\right\rangle^{T}\left(\Phi_{I}-\widetilde{\Phi}_{I}\right)\right\|_{H^{1}, \eta, I} \leq C \eta\|f\|_{H_{\mathrm{per}}^{1}}^{2} .
$$

Thus, for $\eta$ sufficiently small, for a positive constant $C$ independent of $\eta$, we have

$$
\alpha\langle f, f\rangle+\left\langle f, H^{\mathrm{PAW}} f\right\rangle \geq C\|f\|_{H_{\mathrm{per}}^{1}}^{2} .
$$

Since $f$ is a generalized eigenfunction of $H^{\mathrm{PAW}}$, we have

$$
\alpha\langle f, f\rangle+E^{\mathrm{PAW}}\left\langle f, S^{\mathrm{PAW}} f\right\rangle \geq C\|f\|_{H_{\mathrm{per}}^{1}}^{2} .
$$

From item 5 of Lemma B.1, it holds

$$
\left\langle f, S^{\mathrm{PAW}} f\right\rangle \leq\langle f, f\rangle+C \eta\|f\|_{H_{\mathrm{per}}^{1}}^{2},
$$


which yields

$$
C\|f\|_{H_{\mathrm{per}}^{1}}^{2} \leq \alpha\langle f, f\rangle+E^{\mathrm{PAW}}\left\langle f, S^{\mathrm{PAW}} f\right\rangle \leq\left(E^{\mathrm{PAW}}+\alpha\right)\langle f, f\rangle+C \eta E^{\mathrm{PAW}}\|f\|_{H_{\mathrm{per}}^{1}}^{2}
$$

meaning that

$$
\|f\|_{H_{\mathrm{per}}^{1}}^{2} \leq \frac{E^{\mathrm{PAW}}+\alpha}{C-C \eta E^{\mathrm{PAW}}}\langle f, f\rangle,
$$

with $\langle f, f\rangle=1$ and $C-C \eta E^{\mathrm{PAW}}>0$ for $\eta$ small enough.

Proof of the lower bound of Theorem 3.3. Let $f$ be an $L_{\mathrm{per}}^{2}$-normalized eigenfunction associated to the lowest eigenvalue of $H^{\mathrm{PAW}} f=E^{\mathrm{PAW}} S^{\mathrm{PAW}} f$. Then we have:

$$
\begin{aligned}
\left\langle f, H^{\mathrm{PAW}} f\right\rangle & =\langle f, H f\rangle+\sum_{I=\{0, a\}}\left\langle\left\langle\widetilde{p}^{I}, f\right\rangle^{T}\left(\Phi_{I}+\widetilde{\Phi}_{I}\right), H\left\langle\widetilde{p}^{I}, f\right\rangle^{T}\left(\Phi_{I}-\widetilde{\Phi}_{I}\right)\right\rangle \\
& \geq E_{0}\langle f, f\rangle-C \sum_{I=\{0, a\}}\left\|\left\langle\widetilde{p}^{I}, f\right\rangle^{T}\left(\Phi_{I}+\widetilde{\Phi}_{I}\right)\right\|_{H^{1}, \eta, I}\left\|\left\langle\widetilde{p}^{I}, f\right\rangle^{T}\left(\Phi_{I}-\widetilde{\Phi}_{I}\right)\right\|_{H^{1}, \eta, I} \\
& \geq E_{0}\langle f, f\rangle-C \eta\|f\|_{H_{\mathrm{per}}^{1}}^{2},
\end{aligned}
$$

where we used (4.4) in the last inequality.

It remains to show that $\left|\left\langle f, S^{\mathrm{PAW}} f\right\rangle-\langle f, f\rangle\right| \leq C \eta\|f\|_{H_{\mathrm{per}}^{1}}^{2}$ which is precisely item 5 of Lemma B.1. Hence we have

$$
\left\langle f, H^{\mathrm{PAW}} f\right\rangle \geq E_{0}\left\langle f, S^{\mathrm{PAW}} f\right\rangle-C \eta\|f\|_{H_{\mathrm{per}}^{1}}^{2}
$$

We then conclude the proof by Lemma 4.3 .

\subsection{PAW method with pseudopotentials}

In this section, we focus on the truncated equation (2.12) where a pseudopotential is used. First, we see how $H_{\mathrm{ps}}^{\mathrm{PAW}}$ and $H^{\mathrm{VPAW}}$ are related. Recall that $\epsilon$ is the size of the support of the pseudopotential defined in Section 2.2.2.

Lemma 4.4. If $\epsilon \leq \eta$, then

$$
H_{\mathrm{ps}}^{\mathrm{PAW}}=H^{\mathrm{PAW}}+\delta V-\sum_{I \in\{0, a\}}\left(\widetilde{p}^{I}\right)^{T}\left\langle\widetilde{\Phi}_{I}, \delta V \widetilde{\Phi}_{I}^{T}\right\rangle_{I, \eta}\left\langle\widetilde{p}^{I}, \cdot\right\rangle .
$$

where $\delta V=-Z_{0} \chi_{\epsilon}-Z_{a} \chi_{\epsilon}^{a}+Z_{0} \sum_{k \in \mathbb{Z}} \delta_{k}+Z_{a} \sum_{k \in \mathbb{Z}} \delta_{k+a}$.

Proof. By definition of the pseudo wave functions $\widetilde{\phi}_{i}$, we have

$$
\left\langle\phi_{i}^{I}, H \phi_{j}^{I}\right\rangle-\left\langle\widetilde{\phi}_{i}^{I}, H \widetilde{\phi}_{j}^{I}\right\rangle=\left\langle\phi_{i}^{I}, H \phi_{j}^{I}\right\rangle_{I, \eta}-\left\langle\widetilde{\phi}_{i}^{I}, H \widetilde{\phi}_{j}^{I}\right\rangle_{I, \eta} .
$$

By definition of $\delta V, H_{\mathrm{ps}}=H+\delta V$ thus leading to (4.6).

Proposition 4.5. Let $g \in H_{\text {per }}^{1}(0,1)$. Then

$$
\begin{aligned}
\left\langle g, H_{\mathrm{ps}}^{\mathrm{PAW}} g\right\rangle= & \left\langle g, H^{\mathrm{VPAW}} g\right\rangle-2 \sum_{I \in\{0, a\}}\left\langle g-\left\langle\widetilde{p}^{I}, g\right\rangle^{T} \widetilde{\Phi}_{I},\left\langle\widetilde{p}^{I}, g\right\rangle^{T}\left(H \Phi_{I}-(H+\delta V) \widetilde{\Phi}_{I}\right)\right\rangle \\
& +\sum_{I \in\{0, a\}}\left\langle g-\left\langle\widetilde{p}^{I}, g\right\rangle^{T} \widetilde{\Phi}_{I}, \delta V\left(g-\left\langle\widetilde{p}^{I}, g\right\rangle^{T} \widetilde{\Phi}_{I}\right)\right\rangle_{I, \eta} .
\end{aligned}
$$


Proof. By Lemma 4.4, we have

$$
\left\langle g, H_{\mathrm{ps}}^{\mathrm{PAW}} g\right\rangle=\left\langle g, H^{\mathrm{PAW}} g\right\rangle+\langle g, \delta V g\rangle-\sum_{I \in\{0, a\}}\left\langle\left\langle\widetilde{p}^{I}, g\right\rangle^{T} \widetilde{\Phi}_{I}, \delta V\left\langle\widetilde{p}^{I}, g\right\rangle^{T} \widetilde{\Phi}_{I}\right\rangle_{I, \eta}
$$

Applying Proposition 4.1, we obtain

$$
\begin{aligned}
\left\langle g, H_{\mathrm{ps}}^{\mathrm{PAW}} g\right\rangle= & \left\langle g, H^{\mathrm{VPAW}} g\right\rangle-2 \sum_{I \in\{0, a\}}\left\langle g-\left\langle\widetilde{p}^{I}, g\right\rangle^{T} \widetilde{\Phi}_{I},\left\langle\widetilde{p}^{I}, g\right\rangle^{T} H\left(\Phi_{I}-\widetilde{\Phi}_{I}\right)\right\rangle \\
& +\langle g, \delta V g\rangle-\sum_{I \in\{0, a\}}\left\langle\left\langle\widetilde{p}^{I}, g\right\rangle^{T} \widetilde{\Phi}_{I}, \delta V\left\langle\widetilde{p}^{I}, g\right\rangle^{T} \widetilde{\Phi}_{I}\right\rangle .
\end{aligned}
$$

Now, using $H_{\mathrm{ps}}=H+\delta V$, we get

$$
\begin{aligned}
\left\langle g, H_{\mathrm{ps}}^{\mathrm{PAW}} g\right\rangle= & \left\langle g, H^{\mathrm{VPAW}} g\right\rangle-2 \sum_{I \in\{0, a\}}\left\langle g-\left\langle\widetilde{p}^{I}, g\right\rangle^{T} \widetilde{\Phi}_{I},\left\langle\widetilde{p}^{I}, g\right\rangle^{T}\left(H \Phi_{I}-H_{\mathrm{ps}} \widetilde{\Phi}_{I}\right)\right\rangle \\
& -2 \sum_{I \in\{0, a\}}\left\langle g-\left\langle\widetilde{p}^{I}, g\right\rangle^{T} \widetilde{\Phi}_{I}, \delta V\left\langle\widetilde{p}^{I}, g\right\rangle^{T} \widetilde{\Phi}_{I}\right\rangle_{I, \eta} \\
& +\langle g, \delta V g\rangle-\sum_{I \in\{0, a\}}\left\langle\left\langle\widetilde{p}^{I}, g\right\rangle^{T} \widetilde{\Phi}_{I}, \delta V\left\langle\widetilde{p}^{I}, g\right\rangle^{T} \widetilde{\Phi}_{I}\right\rangle .
\end{aligned}
$$

Notice that for each $I$,

$$
\begin{aligned}
-2\langle g- & \left.\left\langle\widetilde{p}^{I}, g\right\rangle^{T} \widetilde{\Phi}_{I}, \delta V\left\langle\widetilde{p}^{I}, g\right\rangle^{T} \widetilde{\Phi}_{I}\right\rangle_{I, \eta}+\langle g, \delta V g\rangle_{I, \eta}-\left\langle\left\langle\widetilde{p}^{I}, g\right\rangle^{T} \widetilde{\Phi}_{I}, \delta V\left\langle\widetilde{p}^{I}, g\right\rangle^{T} \widetilde{\Phi}_{I}\right\rangle \\
& \left.=\langle g, \delta V g\rangle_{I, \eta}-2\left\langle g, \delta V\left\langle\widetilde{p}^{I}, g\right\rangle^{T} \widetilde{\Phi}_{I}\right)\right\rangle_{I, \eta}+\left\langle\left\langle\widetilde{p}^{I}, g\right\rangle^{T} \widetilde{\Phi}_{I}, \delta V\left\langle\widetilde{p}^{I}, g\right\rangle^{T} \widetilde{\Phi}_{I}\right\rangle_{I, \eta} \\
& =\left\langle g-\left\langle\widetilde{p}^{I}, g\right\rangle^{T} \widetilde{\Phi}_{I}, \delta V\left(g-\left\langle\widetilde{p}^{I}, g\right\rangle^{T} \widetilde{\Phi}_{I}\right)\right\rangle_{I, \eta} .
\end{aligned}
$$

Substituting this expression in (4.9), we obtain the expected result.

\subsubsection{Proof of the upper bound of Theorem 3.4}

To prove the upper bound of Theorem 3.4, we proceed as in the proof of the upper bound of Theorem 3.3. We substitute $g$ in Proposition 4.4 by the VPAW eigenfunction $\widetilde{\psi}$ and estimate the difference $\left\langle\widetilde{\psi}, H_{\mathrm{ps}}^{\mathrm{PAW}} \widetilde{\psi}\right\rangle-$ $\left\langle\widetilde{\psi}, H^{\mathrm{VPAW}} \widetilde{\psi}\right\rangle$.

Proof. We start by estimating $\left\langle\widetilde{\psi}, H_{\mathrm{ps}}^{\mathrm{PAW}} \widetilde{\psi}\right\rangle$ where $\widetilde{\psi}$ is the generalized eigenfunction associated to the lowest eigenvalue: $H^{\mathrm{VPAW}} \widetilde{\psi}=E_{0} S^{\mathrm{VPAW}} \widetilde{\psi}$. From Proposition 4.5 we have:

$$
\begin{aligned}
\left\langle\widetilde{\psi}, H_{\mathrm{ps}}^{\mathrm{PAW}} \widetilde{\psi}\right\rangle= & \left\langle\widetilde{\psi}, H^{\mathrm{VPAW}} \widetilde{\psi}\right\rangle-2 \sum_{I \in\{0, a\}}\left\langle\widetilde{\psi}-\left\langle\widetilde{p}^{I}, \widetilde{\psi}\right\rangle^{T} \widetilde{\Phi}_{I},\left\langle\widetilde{p}^{I}, \widetilde{\psi}\right\rangle^{T}\left(H \Phi_{I}-(H+\delta V) \widetilde{\Phi}_{I}\right)\right\rangle \\
& +\sum_{I \in\{0, a\}}\left\langle\widetilde{\psi}-\left\langle\widetilde{p}^{I}, \widetilde{\psi}^{T} \widetilde{\Phi}_{I}, \delta V\left(\widetilde{\psi}-\left\langle\widetilde{p}^{I}, \widetilde{\psi}\right\rangle^{T} \widetilde{\Phi}_{I}\right)\right\rangle_{I, \eta} .\right.
\end{aligned}
$$

The last two terms in the previous equation are estimated separately. 
By equation (A.2), we have for each $I$

$$
\widetilde{\psi}-\left\langle\widetilde{p}^{I}, \widetilde{\psi}\right\rangle^{T} \widetilde{\Phi}_{I}=\psi-\left\langle A_{I}^{-1} p^{I}, \psi\right\rangle^{T} \Phi_{I}
$$

so for each $I$

$$
\begin{aligned}
\left\langle\widetilde{\psi}-\left\langle\widetilde{p}^{I}, \widetilde{\psi}\right\rangle\right. & \left.\cdot \widetilde{\Phi}_{I},\left\langle\widetilde{p}^{I}, \widetilde{\psi}\right\rangle^{T}\left(H \Phi_{I}-(H+\delta V) \widetilde{\Phi}_{I}\right)\right\rangle \\
= & \left\langle\psi-\left\langle A_{I}^{-1} p^{I}, \psi\right\rangle^{T} \Phi_{I},\left\langle\widetilde{p}^{I}, \widetilde{\psi}\right\rangle^{T}\left(H \Phi_{I}-(H+\delta V) \widetilde{\Phi}_{I}\right)\right\rangle \\
= & \left\langle E_{0} \psi-\left\langle A_{I}^{-1} p^{I}, \psi\right\rangle^{T} \mathcal{E}^{I} \Phi_{I},\left\langle\widetilde{p}^{I}, \widetilde{\psi}\right\rangle^{T}\left(\Phi_{I}-\widetilde{\Phi}_{I}\right)\right\rangle \\
& -\left\langle\psi-\left\langle A_{I}^{-1} p^{I}, \psi\right\rangle^{T} \Phi_{I},\left\langle\widetilde{p}^{I}, \widetilde{\psi}^{T} \delta V \widetilde{\Phi}_{I}\right\rangle_{I, \eta} .\right.
\end{aligned}
$$

We have already proved in the proof of the upper bound of Theorem 3.3 that

$$
\left|\left\langle E_{0} \psi-\left\langle A_{I}^{-1} p^{I}, \psi\right\rangle^{T} \mathcal{E}^{I} \Phi_{I},\left\langle\widetilde{p}^{I}, \widetilde{\psi}\right\rangle^{T}\left(\Phi_{I}-\widetilde{\Phi}_{I}\right)\right\rangle\right| \leq C \eta^{2 N} .
$$

Using the parity of the PAW function $\widetilde{\phi}_{k}$ and of the pseudopotential $\chi_{\epsilon}$, it holds

$$
\left\langle\psi-\left\langle A_{I}^{-1} p^{I}, \psi\right\rangle^{T} \Phi_{I},\left\langle\widetilde{p}^{I}, \widetilde{\psi}\right\rangle^{T} \delta V \widetilde{\Phi}_{I}\right\rangle_{I, \eta}=\left\langle\psi_{e}-\left\langle A_{I}^{-1} p^{I}, \psi\right\rangle^{T} \Phi_{I},\left\langle\widetilde{p}^{I}, \widetilde{\psi}\right\rangle^{T} \delta V \widetilde{\Phi}_{I}\right\rangle_{I, \eta},
$$

where $\psi_{e}$ is the even part of $\psi$. By definition of the pseudopotential $\chi_{\epsilon}$, we have for any $f \in L_{\text {per }}^{\infty}(0,1)$

$$
\left|\int_{0}^{1} \chi_{\epsilon}(x) f(x) \mathrm{d} x\right| \leq C\|f\|_{L^{\infty}(-\epsilon, \epsilon)}
$$

Hence

$$
\left|\left\langle\psi-\left\langle A_{I}^{-1} p^{I}, \psi\right\rangle^{T} \Phi_{I},\left\langle\widetilde{p}^{I}, \widetilde{\psi}\right\rangle^{T} \delta V \widetilde{\Phi}_{I}\right\rangle_{I, \eta}\right| \leq C\left\|\psi_{e}-\left\langle A_{I}^{-1} p^{I}, \psi\right\rangle \cdot \Phi_{I}\right\|_{\infty, \eta, I} \|\left\langle\widetilde{p}^{I}, \widetilde{\psi}^{T} \widetilde{\Phi}_{I} \|_{\infty, \eta, I}\right.
$$

From Lemma A.1 and item 3 of Lemma A.3, we obtain

$$
\left|\left\langle\psi-\left\langle A_{I}^{-1} p^{I}, \psi\right\rangle^{T} \Phi_{I},\left\langle\widetilde{p}^{I}, \widetilde{\psi}\right\rangle^{T} \delta V \widetilde{\Phi}_{I}\right\rangle_{I, \eta}\right| \leq C \eta^{2 N}
$$

For the last term in equation (4.10), first notice that by parity of PAW functions the odd part of $\widetilde{\psi}-$ $\left\langle\widetilde{p}^{I}, \widetilde{\psi}\right\rangle^{T} \widetilde{\Phi}_{I}$ is equal to the odd part of $\psi$. Let $\psi_{o}$ be the odd part of $\psi$. Using again Lemma A.1, we obtain

$$
\begin{aligned}
\left|\left\langle\widetilde{\psi}-\left\langle\widetilde{p}^{I}, \widetilde{\psi}\right\rangle^{T} \widetilde{\Phi}_{I}, \delta V\left(\widetilde{\psi}-\left\langle\widetilde{p}^{I}, \widetilde{\psi}\right\rangle^{T} \widetilde{\Phi}_{I}\right)\right\rangle_{I, \eta}\right| & \leq C\left\|\psi_{e}-\left\langle A_{I}^{-1} p^{I}, \psi\right\rangle \cdot \Phi_{I}\right\|_{\infty, \eta, I}^{2}+\int_{-\eta}^{\eta} \chi_{\epsilon}(x)\left|\psi_{o}(x)\right|^{2} \mathrm{~d} x \\
& \leq C \eta^{4 N}+\int_{-\eta}^{\eta} \chi_{\epsilon}(x)\left|\psi_{o}(x)\right|^{2} \mathrm{~d} x .
\end{aligned}
$$


By Lemma 4.2 in [3], we know that for $|x| \leq \eta$, there exists a constant independent of $\eta$ such that:

$$
\left|\psi_{o}(x)\right|^{2} \leq C \eta^{2}
$$

hence

$$
\left|\left\langle\widetilde{\psi}-\left\langle\widetilde{p}^{I}, \widetilde{\psi}\right\rangle^{T} \widetilde{\Phi}_{I}, \delta V\left(\widetilde{\psi}-\left\langle\widetilde{p}^{I}, \widetilde{\psi}\right\rangle^{T} \widetilde{\Phi}_{I}\right)\right\rangle_{I, \eta}\right| \leq C \eta^{2}
$$

Thus

$$
E_{\mathrm{ps}}^{\mathrm{PAW}}\left\langle\widetilde{\psi}, S^{\mathrm{PAW}} \widetilde{\psi}\right\rangle \leq E_{0}\left\langle\widetilde{\psi}, S^{\mathrm{VPAW}} \widetilde{\psi}\right\rangle+C \eta^{2},
$$

and we conclude using item 4 of Lemma B.1.

\subsubsection{Proof of the lower bound of Theorem 3.4}

The core of the proof of the error on the lowest PAW eigenvalue lies on the estimation of $f-\sum_{i=1}^{N}\left\langle\widetilde{p}_{i}, f\right\rangle \widetilde{\phi}_{i}$, which is of the order of the best approximation of $f$ by the family of pseudo wave functions $\left(\widetilde{\phi}_{i}\right)_{1 \leq i \leq N}$. In order to give estimates of the best approximation, we analyze the behavior of the PAW eigenfunction $f$. In this regard, an estimate on the PAW eigenvalue will be useful.

Lemma 4.6. Let $E_{\mathrm{ps}}^{\mathrm{PAW}}$ be the lowest generalized eigenvalue of $(2.12)$. Then $E_{\mathrm{ps}}^{\mathrm{PAW}}$ is bounded from below as $\eta$ goes to 0 .

Proof. Let $f$ be an $L_{\mathrm{per}}^{2}$-normalized generalized eigenfunction of (2.12) associated to $E_{\mathrm{ps}}^{\mathrm{PAW}}$. By (4.5), we have

$$
\alpha\langle f, f\rangle+\left\langle f, H^{\mathrm{PAW}} f\right\rangle \geq C\|f\|_{H_{\mathrm{per}}^{1}}^{2},
$$

where $C$ is some positive constant, $\alpha$ the coercivity constant of $H$ defined by (2.1) and $H^{\mathrm{PAW}}$ the truncated PAW operator (2.10). By Lemma 4.4, we have

$$
\alpha\langle f, f\rangle+\left\langle f, H_{\mathrm{ps}}^{\mathrm{PAW}} f\right\rangle \geq C\|f\|_{H_{\mathrm{per}}^{1}}-\langle f, \delta V f\rangle+\sum_{I \in\{0, a\}}\left\langle\left\langle\widetilde{p}^{I}, f\right\rangle^{T} \widetilde{\Phi}_{I}, \delta V\left\langle\widetilde{p}^{I}, f\right\rangle^{T} \widetilde{\Phi}_{I}\right\rangle .
$$

We have

$$
\begin{aligned}
\left|\langle f, \delta V f\rangle_{0, \eta}\right| & \leq Z_{0}\left|\int_{-\epsilon}^{\epsilon} \chi_{\epsilon}(x)\left(|f(x)|^{2}-|f(0)|^{2}\right) \mathrm{d} x\right| \\
& \leq C \int_{-\epsilon}^{\epsilon} \chi_{\epsilon}(x)|f(x)+f(0)||f(x)-f(0)| \mathrm{d} x \\
& \leq C\|f\|_{\infty, \eta}\|f-f(0)\|_{\infty, \eta} \\
& \leq C \eta^{1 / 2}\|f\|_{H_{\mathrm{per}}^{1}}^{2},
\end{aligned}
$$

where in the second inequality, we used $\int_{-\epsilon}^{\epsilon} \chi_{\epsilon}(x) \mathrm{d} x=1$ and $\epsilon \leq \eta$ and in the last inequality, $\|f-f(0)\|_{\infty, \eta} \leq$ $C \eta^{1 / 2}\|f\|_{H_{\mathrm{per}}^{1}}$ and the Sobolev embedding $\|f\|_{L^{\infty}} \leq C\|f\|_{H_{\mathrm{per}}^{1}}$.

Similarly, we have

$$
\left|\left\langle\left\langle\widetilde{p}^{I}, f\right\rangle^{T} \widetilde{\Phi}_{I}, \delta V\left\langle\widetilde{p}^{I}, f\right\rangle^{T} \widetilde{\Phi}_{I}\right\rangle\right| \leq C \eta^{1 / 2}\left\|\left\langle\widetilde{p}^{I}, f\right\rangle^{T} \widetilde{\Phi}_{I}\right\|_{H_{\mathrm{per}}^{1}}^{2},
$$

thus by items 3 and 4 of Lemma A.3, we obtain

$$
\left|\left\langle\left\langle\widetilde{p}^{I}, f\right\rangle^{T} \widetilde{\Phi}_{I}, \delta V\left\langle\widetilde{p}^{I}, f\right\rangle^{T} \widetilde{\Phi}_{I}\right\rangle\right| \leq C \eta\|f\|_{H_{\mathrm{per}}^{1}}^{2} .
$$


Thus injecting (4.12) and (4.13) in (4.11), we get for $\eta$ sufficiently small and a positive constant $C$,

$$
\alpha\langle f, f\rangle+\left\langle f, H_{\mathrm{ps}}^{\mathrm{PAW}} f\right\rangle \geq C\|f\|_{H_{\mathrm{per}}^{1}}^{2} .
$$

Thus

$$
\alpha\langle f, f\rangle+E_{\mathrm{ps}}^{\mathrm{PAW}}\left\langle f, S^{\mathrm{PAW}} f\right\rangle \geq C\|f\|_{H_{\mathrm{per}}^{1}}^{2},
$$

and we conclude the proof using item 5 of Lemma B.1.

Lemma 4.7. Let $f$ be a generalized eigenfunction of (2.12) and $k \in \mathbb{N}^{*}$. Then there exists a constant $C$ independent of $\eta, \epsilon$ and $f$ such that

$$
\left\|f^{(k)}\right\|_{\infty, \eta, I} \leq C\left(\frac{1}{\eta^{k-1}}+\frac{1}{\epsilon^{k-1}}\right)\|f\|_{\infty, \eta, I} .
$$

Proof. This lemma is proved by induction on $k$. We show the lemma for $I=0$ and drop the index $I$.

Base case. To get the desired estimate for $f^{\prime}$, we integrate $(2.12)$ on $(-\eta, x)$ where $x \in(-\eta, \eta)$ :

$$
\begin{gathered}
-f^{\prime \prime}(x)+\frac{1}{\epsilon} \chi\left(\frac{x}{\epsilon}\right) f(x)+\langle\widetilde{p}, f\rangle^{T}\left(\left\langle\Phi, H \Phi^{T}\right\rangle_{\eta}-\left\langle\widetilde{\Phi}, H_{\mathrm{ps}} \widetilde{\Phi}^{T}\right\rangle_{\eta}\right) \widetilde{p}^{I}(x) \\
=E_{\mathrm{ps}}^{\mathrm{PAW}}\left(f(x)+\langle\widetilde{p}, f\rangle^{T}\left(\left\langle\Phi, \Phi^{T}\right\rangle_{\eta}-\left\langle\widetilde{\Phi}, \widetilde{\Phi}^{T}\right\rangle_{\eta}\right) \widetilde{p}(x)\right) .
\end{gathered}
$$

First, we bound $f^{\prime}( \pm \eta)$ and $f^{\prime}(a \pm \eta)$. For $x \in \bigcup_{k \in \mathbb{Z}}(\eta+k, a-\eta+k)$ and $x \in \bigcup_{k \in \mathbb{Z}}(a+\eta+k, 1-\eta+k), f$ satisfies

$$
-f^{\prime \prime}(x)=E_{\mathrm{ps}}^{\mathrm{PAW}} f(x) .
$$

From Section 4.2.1, we already know that

$$
E_{\mathrm{ps}}^{\mathrm{PAW}} \leq E_{0}+C \eta^{2}
$$

Since $E_{0}<0$, then for $\eta$ sufficiently small, $E_{\mathrm{ps}}^{\mathrm{PAW}}<0$. Thus, outside the intervals $(-\eta, \eta)$ and $(a-\eta, a+\eta), f$ can be written as

$$
f(x)=a_{1} \cos h\left(\sqrt{-E_{\mathrm{ps}}^{\mathrm{PAW}}} x\right)+a_{2} \sin h\left(\sqrt{-E_{\mathrm{ps}}^{\mathrm{PAW}}} x\right) .
$$

The coefficients $a_{1}$ and $a_{2}$ are determined by the continuity of $f$ at $\pm \eta$ and $a \pm \eta$. By Lemma $4.6, E_{\mathrm{ps}}^{\mathrm{PAW}}$ is bounded from below as $\eta$ goes to 0 , hence $\left|f^{\prime}( \pm \eta)\right|$ and $f^{\prime}(a \pm \eta)$ are uniformly bounded with respect to $\eta$ as $\eta$ goes to 0 .

We now prove that $f^{\prime}(x)$ is uniformly bounded with respect to $\eta$ and $\epsilon$ as $\eta, \epsilon \rightarrow 0$ for $x \in \bigcup_{k \in \mathbb{Z}}(-\eta+k, \eta+k)$ and $x \in \bigcup_{k \in \mathbb{Z}}(a-\eta+k, a+\eta+k) \cdot \chi(\dot{\bar{\epsilon}})$ is a bounded function supported in $(-\epsilon, \epsilon)$, we have

$$
\left|\frac{1}{\epsilon} \int_{-\eta}^{x} \chi\left(\frac{t}{\epsilon}\right) f(t) \mathrm{d} t\right| \leq C\|f\|_{\infty, \eta}
$$

To finish the proof, it suffices to show that the remaining terms are at most of order $\mathcal{O}\left(\frac{\|f\|_{\infty, \eta}}{\eta}\right)$ with respect to the $\infty$-norm. These terms will be treated separately.

(1) For $\langle\widetilde{p}, f\rangle^{T}\left\langle\Phi, \Phi^{T}\right\rangle_{\eta} \widetilde{p}(x)$, by item 2 of Lemma A.2, we have

$$
\begin{aligned}
\langle\widetilde{p}, f\rangle^{T}\left\langle\Phi, \Phi^{T}\right\rangle_{\eta} \widetilde{p}(x)= & \left(M_{\eta} \int_{-1}^{1} \rho(t) f(\eta t) P(t) \mathrm{d} t\right)^{T} \\
& \times\left\langle\left(\frac{C_{1}^{-1}}{0}\right) \Phi, \Phi^{T}\left(C_{1}^{-T} \mid 0\right)\right\rangle_{\eta} M_{\eta} \rho\left(\frac{x}{\eta}\right) P\left(\frac{x}{\eta}\right)
\end{aligned}
$$


According to item 3 of Lemma A.2, we already know that

$$
\left\|\left(\frac{C_{1}^{-1}}{0}\right) \Phi\right\|_{\infty, \eta} \leq C
$$

thus

$$
\left|\langle\widetilde{p}, f\rangle^{T}\left\langle\Phi, \Phi^{T}\right\rangle_{\eta} \widetilde{p}(x)\right| \leq C\|f\|_{\infty, \eta}\left|\rho\left(\frac{x}{\eta}\right) P\left(\frac{x}{\eta}\right)\right| .
$$

(2) Using item 2 of Lemma A.2, the term $\langle\widetilde{p}, f\rangle^{T}\left\langle\widetilde{\Phi}, \widetilde{\Phi}^{T}\right\rangle_{\eta} \widetilde{p}(x)$ can be written as

$$
\langle\widetilde{p}, f\rangle^{T}\left\langle\widetilde{\Phi}, \widetilde{\Phi}^{T}\right\rangle_{\eta} \widetilde{p}(x)=\left(M_{\eta} \int_{-1}^{1} \rho(t) f(\eta t) P(t) \mathrm{d} t\right)^{T}\left\langle P(\dot{\bar{\eta}}), P^{T}(\dot{\bar{\eta}})\right\rangle_{\eta} M_{\eta} \rho\left(\frac{x}{\eta}\right) P\left(\frac{x}{\eta}\right) .
$$

Hence, we obtain

$$
\left|\langle\widetilde{p}, f\rangle^{T}\left\langle\widetilde{\Phi}, \widetilde{\Phi}^{T}\right\rangle_{\eta} \widetilde{p}^{I}(x)\right| \leq C\|f\|_{\infty, \eta}\left|\rho\left(\frac{x}{\eta}\right) P\left(\frac{x}{\eta}\right)\right| \cdot
$$

(3) On the LHS of (4.16), the term $\langle\widetilde{p}, f\rangle^{T}\left\langle\Phi, H \Phi^{T}\right\rangle_{\eta} \widetilde{p}(x)$ is given by

$$
\langle\widetilde{p}, f\rangle^{T}\left\langle\Phi, H \Phi^{T}\right\rangle_{\eta} \widetilde{p}(x)=\langle\widetilde{p}, f\rangle^{T}\left\langle\Phi^{\prime}, \Phi^{\prime T}\right\rangle_{\eta} \widetilde{p}(x)-Z_{0}\langle\widetilde{p}, f\rangle^{T} \Phi(0) \Phi(0)^{T} \widetilde{p}(x) .
$$

Similarly to item 1 above, we can show that

$$
\left|\langle\widetilde{p}, f\rangle^{T} \Phi(0) \Phi(0)^{T} \widetilde{p}(x)\right| \leq C\|f\|_{\infty, \eta}\left|\rho\left(\frac{x}{\eta}\right) P\left(\frac{x}{\eta}\right)\right| .
$$

Using item 3 of Lemma A.2,

$$
\left\|\left(\frac{C_{1}^{-1}}{0}\right) \Phi^{\prime}\right\|_{\infty, \eta} \leq \frac{C}{\eta}
$$

we get

$$
\left|\langle\widetilde{p}, f\rangle^{T}\left\langle\Phi^{\prime}, \Phi^{\prime T}\right\rangle_{\eta} \widetilde{p}(x)\right| \leq \frac{C}{\eta}\|f\|_{\infty, \eta}\left|\rho\left(\frac{x}{\eta}\right) P\left(\frac{x}{\eta}\right)\right|
$$

(4) Finally, for $\langle\widetilde{p}, f\rangle^{T}\left\langle\widetilde{\Phi}, H_{\mathrm{ps}} \widetilde{\Phi}^{T}\right\rangle_{\eta} \widetilde{p}(x)$, we have

$$
\langle\widetilde{p}, f\rangle^{T}\left\langle\widetilde{\Phi}, H_{\mathrm{ps}} \widetilde{\Phi}^{T}\right\rangle_{\eta} \widetilde{p}(x)=\langle\widetilde{p}, f\rangle^{T}\left\langle\widetilde{\Phi}^{\prime}, \widetilde{\Phi}^{\prime T}\right\rangle_{\eta} \widetilde{p}(x)-\frac{Z_{0}}{\epsilon}\langle\widetilde{p}, f\rangle^{T} \int_{-\epsilon}^{\epsilon} \chi\left(\frac{t}{\epsilon}\right) P\left(\frac{t}{\eta}\right) P\left(\frac{t}{\eta}\right)^{T} \mathrm{~d} t \widetilde{p}(x) .
$$

Since $\epsilon \leq \eta,\left|\int_{-\epsilon}^{\epsilon} \chi\left(\frac{t}{\epsilon}\right) P\left(\frac{t}{\eta}\right) P\left(\frac{t}{\eta}\right)^{T} \mathrm{~d} t\right| \leq C \epsilon$ where $C$ is independent of $\eta$ and $\epsilon$. Moreover,

$$
\langle\widetilde{p}, f\rangle^{T}\left\langle\widetilde{\Phi}^{\prime}, \widetilde{\Phi}^{\prime T}\right\rangle_{\eta} \widetilde{p}(x)=\frac{1}{\eta^{2}}\left(M_{\eta} \int_{-1}^{1} \rho(t) f(\eta t) P(t) \mathrm{d} t\right)^{T}\left\langle P^{\prime}(\dot{\bar{\eta}}), P^{\prime}(\dot{\dot{\eta}})^{T}\right\rangle_{\eta} M_{\eta} \rho\left(\frac{x}{\eta}\right) P\left(\frac{x}{\eta}\right),
$$

hence

$$
\left|\langle\widetilde{p}, f\rangle^{T}\left\langle\widetilde{\Phi}^{\prime}, \widetilde{\Phi}^{\prime T}\right\rangle_{\eta} \widetilde{p}(x)\right| \leq \frac{C}{\eta}\|f\|_{\infty, \eta}\left|\rho\left(\frac{x}{\eta}\right) P\left(\frac{x}{\eta}\right)\right| .
$$


Inductive step. Suppose the statement is true for any $k \leq n$. We differentiate (4.16) $(n-1)$ times

$$
\begin{gathered}
-f^{(n+1)}(x)+\frac{1}{\epsilon}(\chi(\dot{\bar{\epsilon}}) f)^{(n-1)}(x)+\langle\widetilde{p}, f\rangle^{T}\left(\left\langle\Phi, H \Phi^{T}\right\rangle_{\eta}-\left\langle\widetilde{\Phi}, H_{\mathrm{ps}} \widetilde{\Phi}^{T}\right\rangle_{\eta}\right) \widetilde{p}^{(n-1)}(x) \\
=E_{\mathrm{ps}}^{\mathrm{PAW}}\left(f^{(n-1)}(x)+\langle\widetilde{p}, f\rangle^{T}\left(\left\langle\Phi, \Phi^{T}\right\rangle_{\eta}-\left\langle\widetilde{\Phi}, \widetilde{\Phi}^{T}\right\rangle_{\eta}\right) \widetilde{p}^{(n-1)}(x)\right) .
\end{gathered}
$$

By the induction hypothesis and since $\epsilon \leq \eta$, we have

$$
\left|\frac{1}{\epsilon}(\chi(\dot{\bar{\epsilon}}) f)^{(n-1)}(x)\right| \leq C\left(\frac{\|f\|_{\infty, \eta}}{\epsilon^{n}}+\sum_{k=1}^{n-1} \frac{\left\|f^{(k)}\right\|_{\infty, \eta}}{\epsilon^{n-k}}\right) \leq C \frac{\|f\|_{\infty, \eta}}{\epsilon^{n}} .
$$

We simply give an estimate of the term

$$
\langle\widetilde{p}, f\rangle^{T}\left\langle\Phi, H \Phi^{T}\right\rangle_{\eta} \widetilde{p}^{(n-1)}(x),
$$

since the other terms appearing in (4.19) can be treated the same way. By (4.17), we already know that

$$
\left|\langle\widetilde{p}, f\rangle^{T} \Phi(0) \Phi(0)^{T} \widetilde{p}^{(n-1)}(x)\right| \leq \frac{C}{\eta^{n-1}}\|f\|_{\infty, \eta}\left|(\rho P)^{(n-1)}\left(\frac{x}{\eta}\right)\right| \leq \frac{C}{\eta^{n-1}}\|f\|_{\infty, \eta} .
$$

By (4.18), we have

$$
\left|\langle\widetilde{p}, f\rangle^{T}\left\langle\Phi^{\prime}, \Phi^{\prime T}\right\rangle_{\eta} \widetilde{p}^{(n-1)}(x)\right| \leq \frac{C}{\eta^{n}}\|f\|_{\infty, \eta}\left|(\rho P)^{(n-1)}\left(\frac{x}{\eta}\right)\right| \leq \frac{C}{\eta^{n}}\|f\|_{\infty, \eta} .
$$

Injecting (4.20) and (4.21) in (4.19) concludes the proof.

We next provide an estimation of the best approximation by $\left(\widetilde{\phi}_{i}\right)_{1 \leq i \leq N}$ of the even part $f_{e}$ of the PAW eigenfunction $f$.

Lemma 4.8. Let $f$ be an eigenfunction associated to the lowest eigenvalue of (2.12) and let $f_{e}$ be the even part of $f$. Suppose that $\epsilon \leq \eta$. Then there exists a family of coefficients $\left(\alpha_{i}\right)_{1 \leq i \leq N}$ and $C$ independent of $\eta$ and $\epsilon$ such that

$$
\left\|f_{e}-\sum_{i=1}^{N} \alpha_{i} \widetilde{\phi}_{i}^{I}\right\|_{\infty, \eta, I} \leq C \eta\left(\frac{\eta}{\epsilon}\right)^{2 N-1}\|f\|_{\infty, \eta, I}
$$

and for the same family of coefficients

$$
\left\|f_{e}^{\prime}-\sum_{i=1}^{N} \alpha_{i} \widetilde{\phi}_{i}^{I \prime}\right\|_{\infty, \eta, I} \leq C\left(\frac{\eta}{\epsilon}\right)^{2 N}\|f\|_{\infty, \eta, I}
$$

Proof. For clarity, we will drop the index $I$ in this proof. First we write the Taylor expansion of $f$ around 0 , for $|x| \leq \eta$ :

$$
f_{e}(x)=\sum_{k=0}^{N-1} \frac{f^{(2 k)}(0)}{(2 k) !} x^{2 k}+R_{2 N}(f)(x),
$$

where $R_{2 N}(f)$ is the integral form of the remainder

$$
R_{2 N}(f)(x)=\int_{0}^{x} \frac{f^{(2 N)}(t)}{(2 N-1) !}(x-t)^{2 N-1} \mathrm{~d} t .
$$


The remainder $R_{2 N}(f)$ satisfies

$$
\begin{aligned}
\left|R_{2 N}(f)(x)\right| & \leq C \eta^{2 N}\left\|f^{(2 N)}\right\|_{\infty, \eta} \\
& \leq C \eta\left(\frac{\eta}{\epsilon}\right)^{2 N-1}\|f\|_{\infty, \eta},
\end{aligned}
$$

where, in the second inequality, we used Lemma 4.7. Thus, the best approximation of $f$ by a linear combination of $\left(\widetilde{\phi}_{k}\right)_{1 \leq k \leq N}$ is at most of order $\eta$. In the remainder of the proof, we will show that this order is attainable. Setting $t=\frac{x}{\eta}$, we obtain

$$
f_{e}(x)-\sum_{i=1}^{N} \alpha_{i} \widetilde{\phi}_{i}(x)=\sum_{k=0}^{N-1} \frac{f^{(2 k)}(0)}{(2 k) !} \eta^{2 k} t^{2 k}-\sum_{i=1}^{N} \alpha_{i} \widetilde{\phi}_{i}(\eta t)+R_{2 N}(f)(\eta t) .
$$

By Lemma 4.7, we have for all $1 \leq k \leq N-1$ :

$$
\left|\frac{f^{(2 k)}(0)}{(2 k) !} \eta^{2 k}\right| \leq C \eta\left(\frac{\eta}{\epsilon}\right)^{2 k-1}
$$

The family $\left(\widetilde{\phi}_{j}\right)_{1 \leq j \leq N}$ satisfies

$$
\widetilde{\Phi}(x)=C_{\eta}^{(P)} P\left(\frac{x}{\eta}\right),
$$

where $P(t)$ is the vector of polynomials $P_{k}(t)=\frac{1}{2^{k} k !}\left(t^{2}-1\right)^{k}$. By Lemma 4.9 in [3], we know that $C_{\eta}^{(P)}$ can be written:

$$
C_{\eta}^{(P)}=\Phi(\eta) e_{0}^{T}+\eta \Phi^{\prime}(\eta) \beta_{1}^{T}+\mathcal{O}\left(\eta^{2}\right),
$$

where $\beta_{1}$ is a vector of $\mathbb{R}^{d}$ uniformly bounded in $\eta$. Thus we have

$$
\sum_{k=0}^{N-1} \frac{f^{(2 k)}(0)}{(2 k) !} \eta^{2 k} t^{2 k}-\sum_{i=1}^{N} \alpha_{i} \widetilde{\phi}_{i}(\eta t)=f(0)-\alpha^{T} \Phi(\eta)+\mathcal{O}\left(\eta\left(\frac{\eta}{\epsilon}\right)^{2 N-1}\right) .
$$

To get the result, $\alpha$ has to be chosen such that $\alpha^{T} \Phi(\eta)=f(0)$, which is possible because $\Phi(\eta) \neq 0$.

For $f_{e}^{\prime}$, we proceed the same way. However, by Lemma 4.7, the remainder of the Taylor expansion of $f_{e}^{\prime}$ satisfies

$$
\left|R_{2 N}\left(f^{\prime}\right)(x)\right| \leq C \eta^{2 N}\left\|f^{(2 N+1)}\right\|_{\infty, \eta} \leq C\left(\frac{\eta}{\epsilon}\right)^{2 N}\|f\|_{\infty, \eta} .
$$

We simply have to check that $\left\|\widetilde{\Phi}^{\prime}\right\|_{\infty, \eta}$ is bounded when $\eta$ goes to 0 . By $(4.22)$ and because $P_{0}^{\prime}=0$,

$$
\widetilde{\Phi}^{\prime}(x)=\Phi^{\prime}(\eta) \beta_{1}^{T} P^{\prime}\left(\frac{x}{\eta}\right)+\mathcal{O}(\eta),
$$

hence $\left\|\widetilde{\Phi}^{\prime}\right\|_{\infty, \eta}$ is bounded when $\eta$ goes to 0 .

We can now give an estimate for $f_{e}-\sum_{i=1}^{N}\langle\widetilde{p}, f\rangle \phi_{i}$.

Lemma 4.9. Assume that $f$ is the generalized eigenfunction of (2.12) associated to the lowest generalized eigenvalue. Let $f_{e}$ be the even part of $f$. Then

$$
\left\|f_{e}-\left\langle\widetilde{p}^{I}, f\right\rangle^{T} \widetilde{\Phi}_{I}\right\|_{\infty, \eta, I} \leq C \eta\left(\frac{\eta}{\epsilon}\right)^{2 N-1}\|f\|_{\infty, \eta, I},
$$

and

$$
\left\|f_{e}^{\prime}-\left\langle\widetilde{p}^{I}, f\right\rangle^{T} \widetilde{\Phi}_{I}^{\prime}\right\|_{\infty, \eta, I} \leq C\left(\frac{\eta}{\epsilon}\right)^{2 N-1}\|f\|_{\infty, \eta, I} .
$$


Proof. For clarity, we will drop the index $I$. For any family $\left(\alpha_{j}\right)_{1 \leq j \leq N}$, we have for $x \in(-\eta, \eta)$

$$
\begin{aligned}
f_{e}(x)-\langle\widetilde{p}, f\rangle^{T} \widetilde{\Phi}(x) & =f_{e}(x)-\left\langle\widetilde{p}, f_{e}-\sum_{j=1}^{N} \alpha_{j} \widetilde{\phi}_{j}+\sum_{j=1}^{N} \alpha_{j} \widetilde{\phi}_{j}\right\rangle^{T} \widetilde{\Phi}(x) \\
& =f_{e}(x)-\sum_{j=1}^{N} \alpha_{j} \widetilde{\phi}_{j}-\left\langle\widetilde{p}, f_{e}-\sum_{j=1}^{N} \alpha_{j} \widetilde{\phi}_{j}\right\rangle^{T} \widetilde{\Phi}(x) .
\end{aligned}
$$

By Lemma 4.8, $\left(\alpha_{j}\right)_{1 \leq j \leq N}$ can be chosen such that for any $x \in(-\eta, \eta)$

$$
\left|f_{e}(x)-\sum_{j=1}^{N} \alpha_{j} \widetilde{\phi}_{j}(x)\right| \leq C \eta\left(\frac{\eta}{\epsilon}\right)^{2 N-1}\|f\|_{\infty, \eta} .
$$

Thus by item 3 of Lemma A.3,

$$
\left\|f_{e}-\langle\widetilde{p}, f\rangle^{T} \widetilde{\Phi}\right\|_{\infty, \eta} \leq C \eta\left(\frac{\eta}{\epsilon}\right)^{2 N-1}\|f\|_{\infty, \eta} .
$$

Similarly, we have by item4 of Lemma A.3 for any function $g \in H_{\text {per }}^{1}(0,1)$ with $g^{\prime} \in L^{\infty}(-\eta, \eta)$,

$$
\left|\langle\widetilde{p}, g\rangle^{T} \widetilde{\Phi}^{\prime}(x)\right| \leq C\|g\|_{H^{1}, \eta} \leq C \eta^{1 / 2}\left(\|g\|_{\infty, \eta}+\left\|g^{\prime}\right\|_{\infty, \eta}\right),
$$

and with the same coefficients $\left(\alpha_{j}\right)$,

$$
\left|f_{e}^{\prime}(x)-\sum_{j=1}^{N} \alpha_{j} \widetilde{\phi}_{j}^{\prime}\right| \leq C\left(\frac{\eta}{\epsilon}\right)^{2 N-1}\|f\|_{\infty, \eta} .
$$

So,

$$
\begin{aligned}
\left\|f_{e}^{\prime}-\langle\widetilde{p}, f\rangle^{T} \widetilde{\Phi}^{\prime}\right\|_{\infty, \eta} & \leq\left\|f_{e}^{\prime}-\sum_{j=1}^{N} \alpha_{j} \widetilde{\phi}_{j}^{\prime}\right\|_{\infty, \eta}+\left\|\left\langle\widetilde{p}, f_{e}-\sum_{j=1}^{N} \alpha_{j} \widetilde{\phi}_{j}\right\rangle^{T} \widetilde{\Phi}^{\prime}\right\|_{\infty, \eta} \\
& \leq C\left(\frac{\eta}{\epsilon}\right)^{2 N-1}\|f\|_{\infty, \eta},
\end{aligned}
$$

where in the last inequality, we used (4.23) with Lemma 4.8.

In the proof of the lower bound of Theorem 3.4, we will need to bound terms of the form $\left\|f_{e}-\left\langle\widetilde{p}^{I}, f\right\rangle^{T} \widetilde{\Phi}_{I}\right\|_{\infty, \eta, I}$. If $\epsilon<\eta$, we will get poorer bounds than by setting $\epsilon=\eta$. Hence, from now on, we fix $\epsilon=\eta$.

To estimate the term $\left\langle f-\left\langle\widetilde{p}^{I}, f\right\rangle^{T} \widetilde{\Phi}_{I}, \delta V\left(f-\left\langle\widetilde{p}^{I}, f\right\rangle^{T} \widetilde{\Phi}_{I}\right)\right\rangle_{I, \eta}$, we will need the following estimates.

Lemma 4.10. Let $f$ be an eigenfunction associated to the lowest generalized eigenvalue of (2.12). Then

$$
\left\|f-\left\langle\widetilde{p}^{I}, f\right\rangle^{T} \widetilde{\Phi}_{I}\right\|_{\infty, \eta, I} \leq C \eta\|f\|_{\infty, \eta, I},
$$

and

$$
\left\|f^{\prime}-\left\langle\widetilde{p}^{I}, f\right\rangle^{T} \widetilde{\Phi}_{I}^{\prime}\right\|_{\infty, \eta, I} \leq C\|f\|_{\infty, \eta, I}
$$


Proof. This follows from Lemma 4.9 and that the odd part of $f$ is bounded in $(-\eta, \eta)$ by $\eta\left\|f^{\prime}\right\|_{L^{\infty}(-\eta, \eta)}$, which is itself bounded by $C \eta\|f\|_{L^{\infty}(-\eta, \eta)}$ according to Lemma 4.7 .

We need a uniform bound in $\eta$ on the PAW eigenfunction $f$, in order to prove Theorem 3.4.

Lemma 4.11. Let $f$ be an $L_{\mathrm{per}}^{2}$-normalized eigenfunctions associated to the first eigenvalue of (2.12). Then there exists a positive constant $C$ independent of $\eta$ such that for all $0<\eta \leq \eta_{0}$

$$
\|f\|_{H_{\mathrm{per}}^{1}} \leq C .
$$

Proof. This is a direct consequence of equation (4.14).

We now have all the elements to complete the proof of Theorem 3.4.

Proof of the lower bound in Theorem 3.4. Let $f$ be an $L_{\mathrm{per}}^{2}$-normalized generalized eigenfunction of the PAW eigenvalue problem (2.12). By Proposition 4.5, we have

$$
\begin{aligned}
\left\langle f, H_{\mathrm{ps}}^{\mathrm{PAW}} f\right\rangle= & \left\langle f, H^{\mathrm{VPAW}} f\right\rangle-2 \sum_{I \in\{0, a\}}\left\langle f-\left\langle\widetilde{p}^{I}, f\right\rangle^{T} \widetilde{\Phi}_{I},\left\langle\widetilde{p}^{I}, f\right\rangle^{T}\left(H \Phi_{I}-(H+\delta V) \widetilde{\Phi}_{I}\right)\right\rangle \\
& +\sum_{I \in\{0, a\}}\left\langle f-\left\langle\widetilde{p}^{I}, f\right\rangle^{T} \widetilde{\Phi}_{I}, \delta V\left(f-\left\langle\widetilde{p}^{I}, f\right\rangle^{T} \widetilde{\Phi}_{I}\right)\right\rangle_{\eta, I} .
\end{aligned}
$$

We simply bound terms with $I=0$ as the terms with $I=a$ are treated exactly the same way. First, we estimate $\left\langle f-\langle\widetilde{p}, f\rangle^{T} \widetilde{\Phi}, \delta V\left(f-\langle\widetilde{p}, f\rangle^{T} \widetilde{\Phi}\right)\right\rangle_{I, \eta}$. By Lemma 4.9, we have:

$$
\begin{aligned}
\mid\langle f & \left.-\langle\widetilde{p}, f\rangle^{T} \widetilde{\Phi}, \delta V\left(f-\langle\widetilde{p}, f\rangle^{T} \widetilde{\Phi}\right)\right\rangle_{\eta} \mid \\
& =Z_{0}\left|\left(f(0)-\langle\widetilde{p}, f\rangle^{T} \widetilde{\Phi}(0)\right)^{2}-\int_{-\eta}^{\eta} \chi_{\eta}(x)\left(f(x)-\langle\widetilde{p}, f\rangle^{T} \widetilde{\Phi}(x)\right)^{2} \mathrm{~d} x\right| \\
& =Z_{0}\left|\int_{-\eta}^{\eta} \chi_{\eta}(x)\left(\left(f(x)-\langle\widetilde{p}, f\rangle^{T} \widetilde{\Phi}(x)\right)^{2}-\left(f(0)-\langle\widetilde{p}, f\rangle^{T} \widetilde{\Phi}(0)\right)^{2}\right) \mathrm{d} x\right| \\
& \leq C \eta\left\|f^{\prime}-\langle\widetilde{p}, f\rangle^{T} \widetilde{\Phi}^{\prime}\right\|_{\infty, \eta}\left\|f-\langle\widetilde{p}, f\rangle^{T} \widetilde{\Phi}\right\|_{\infty, \eta} \\
& \leq C \eta^{2}\|f\|_{\infty, \eta}^{2},
\end{aligned}
$$

where in the last inequality, we applied Lemma 4.10.

We then estimate $\left\langle f^{\prime}-\langle\widetilde{p}, f\rangle^{T} \widetilde{\Phi}^{\prime},\langle\widetilde{p}, f\rangle^{T}\left(\Phi^{\prime}-\widetilde{\Phi}^{\prime}\right)\right\rangle$ :

$$
\begin{aligned}
\mid\left\langle f^{\prime}\right. & \left.-\langle\widetilde{p}, f\rangle^{T} \widetilde{\Phi}^{\prime},\langle\widetilde{p}, f\rangle^{T}\left(\Phi^{\prime}-\widetilde{\Phi}^{\prime}\right)\right\rangle \mid \\
& =\left|\int_{-\eta}^{\eta}\left(f_{e}^{\prime}(x)-\langle\widetilde{p}, f\rangle^{T} \widetilde{\Phi}^{\prime}(x)\right)\langle\widetilde{p}, f\rangle^{T}\left(\Phi^{\prime}-\widetilde{\Phi}^{\prime}\right)(x) \mathrm{d} x\right| \\
& \leq C \eta\left\|f_{e}^{\prime}-\langle\widetilde{p}, f\rangle^{T} \widetilde{\Phi}^{\prime}\right\|_{\infty, \eta}\|f\|_{H_{\mathrm{per}}^{1}} \\
& \leq C \eta\|f\|_{\infty, \eta}\|f\|_{H_{\mathrm{per}}^{1}},
\end{aligned}
$$


where in the first inequality, we used item 1 of Lemma A.3 and in the second, Lemma 4.9. Finally, it remains to estimate $\left\langle f-\langle\widetilde{p}, f\rangle^{T} \widetilde{\Phi},\langle\widetilde{p}, f\rangle^{T}\left(\delta_{0} \Phi-\chi_{\eta} \widetilde{\Phi}\right)\right\rangle$ :

$$
\begin{aligned}
\mid\langle f- & \left.\langle\widetilde{p}, f\rangle^{T} \widetilde{\Phi},\langle\widetilde{p}, f\rangle^{T}\left(\delta_{0} \Phi-\chi_{\eta} \widetilde{\Phi}\right)\right\rangle \mid \\
\leq & \left|\left(f(0)-\langle\widetilde{p}, f\rangle^{T} \widetilde{\Phi}(0)\right)\langle\widetilde{p}, f\rangle^{T}(\Phi(0)-\widetilde{\Phi}(0))\right| \\
& +\left|\int_{-\eta}^{\eta} \chi_{\eta}(x)\left(\left(f_{e}(x)-\langle\widetilde{p}, f\rangle^{T} \widetilde{\Phi}(x)\right)\langle\widetilde{p}, f\rangle^{T} \widetilde{\Phi}(x)-\left(f_{e}(0)-\langle\widetilde{p}, f\rangle^{T} \widetilde{\Phi}(0)\right)\langle\widetilde{p}, f\rangle^{T} \widetilde{\Phi}(0)\right) \mathrm{d} x\right| \\
\leq & C \eta^{2}\|f\|_{\infty, \eta}\|f\|_{H_{\mathrm{per}}^{1}}+C \eta\left\|\left(\left(f_{e}-\langle\widetilde{p}, f\rangle^{T} \widetilde{\Phi}\right)\langle\widetilde{p}, f\rangle^{T} \widetilde{\Phi}\right)^{\prime}\right\|_{\infty, \eta} .
\end{aligned}
$$

We have

$$
\begin{aligned}
& \left\|\left(\left(f_{e}-\langle\widetilde{p}, f\rangle^{T} \widetilde{\Phi}\right)\langle\widetilde{p}, f\rangle^{T} \widetilde{\Phi}\right)^{\prime}\right\|_{\infty, \eta} \\
& \quad \leq\left\|\left(f_{e}-\langle\widetilde{p}, f\rangle^{T} \widetilde{\Phi}\right)\langle\widetilde{p}, f\rangle^{T} \widetilde{\Phi}^{\prime}\right\|_{\infty, \eta}+\left\|\left(f_{e}^{\prime}-\langle\widetilde{p}, f\rangle^{T} \widetilde{\Phi}^{\prime}\right)\langle\widetilde{p}, f\rangle^{T} \widetilde{\Phi}\right\|_{\infty, \eta} \\
& \quad \leq C \eta\|f\|_{\infty, \eta}\|f\|_{H_{\mathrm{per}}^{1}}+C\|f\|_{\infty, \eta}\|f\|_{H_{\mathrm{per}}^{1}}
\end{aligned}
$$

where we applied Lemma 4.9 and items 3 and 4 of Lemma A.3. Thus,

$$
\left|\left\langle f-\langle\widetilde{p}, f\rangle^{T} \widetilde{\Phi},\langle\widetilde{p}, f\rangle^{T}\left(\delta_{0} \Phi-\chi_{\eta} \widetilde{\Phi}\right)\right\rangle\right| \leq C \eta\|f\|_{\infty, \eta}\|f\|_{H_{\mathrm{per}}^{1}}
$$

Inserting (4.25)-(4.27), in (4.24), we obtain

$$
\begin{aligned}
\left\langle f, H_{\mathrm{ps}}^{\mathrm{PAW}} f\right\rangle & \geq\left\langle f, H^{\mathrm{VPAW}} f\right\rangle-C \eta^{2}\|f\|_{L^{\infty}}^{2}-C \eta\|f\|_{\infty, \eta}\|f\|_{H_{\mathrm{per}}^{1}} \\
& \geq E_{0}\left\langle f, S^{\mathrm{VPAW}} f\right\rangle-C \eta^{2}\|f\|_{L^{\infty}}^{2}-C \eta\|f\|_{\infty, \eta}\|f\|_{H_{\mathrm{per}}^{1}} .
\end{aligned}
$$

Using item 3 of Lemma B.1, we obtain

$$
\begin{aligned}
E_{0}\left\langle f, S^{\mathrm{PAW}} f\right\rangle-C \eta^{2}\|f\|_{L^{\infty}}^{2}-C \eta\|f\|_{\infty, \eta}\|f\|_{H_{\mathrm{per}}^{1}} & \leq\left\langle f, H_{\mathrm{ps}}^{\mathrm{PAW}} f\right\rangle \\
& \leq E_{\mathrm{ps}}^{\mathrm{PAW}}\left\langle f, S^{\mathrm{PAW}} f\right\rangle,
\end{aligned}
$$

and the result follows from Lemma 4.11 and the Sobolev embedding $\|f\|_{L^{\infty}} \leq C\|f\|_{H_{\mathrm{per}}^{1}}$.

\subsubsection{The PAW method with odd functions and proof of Theorem 3.5}

The poor upper bound of Theorem 3.4 is due to the poor approximation of $f$ by the pseudo wave functions $\widetilde{\phi}_{k}$. The latter are only even polynomials inside the cut-off region, hence incorporating odd functions to the PAW treatment should improve the upper bound on the PAW eigenvalue $E_{\mathrm{ps}}^{\mathrm{PAW}}$.

The odd atomic wave functions are the functions

$$
\tilde{\theta}_{k}(x)=\sin (2 \pi k x), \quad k \in \mathbb{N}^{*},
$$

which are eigenfunctions of the atomic Hamiltonian $-\frac{\mathrm{d}^{2}}{\mathrm{~d} x^{2}}-Z_{0} \sum_{k \in \mathbb{Z}} \delta_{k}$. As these functions are already smooth, there is no need to take pseudo wave functions different from the atomic wave functions.

To define the corresponding projector functions $\widetilde{q}_{k}$, we consider

$$
G=\left(\int_{-\eta}^{\eta} \rho_{\eta}(t) \sin (2 \pi j t) \sin (2 \pi k t) \mathrm{d} t\right)_{1 \leq j, k \leq N},
$$


where $\rho_{\eta}$ is the smooth cut-off function defined in Section 2.4. $G$ is an invertible matrix since it is the Gram matrix of the linearly independent family of functions $(\sin (2 \pi k x))_{1 \leq k \leq N}$. Now let $\widetilde{q}_{k}$ be defined by

$$
\widetilde{q}_{k}(x)=\rho_{\eta}(x) \sum_{j=1}^{N}\left(G^{-1}\right)_{j k} \widetilde{\theta}_{j}(x),
$$

so the functions $\left(\widetilde{\theta}_{k}\right)_{1 \leq k \leq N}$ and $\left(\widetilde{q}_{k}\right)_{1 \leq k \leq N}$ satisfy

$$
\left\langle\widetilde{q}_{j}, \widetilde{\theta}_{k}\right\rangle=\delta_{j k}
$$

The functions $\left(\widetilde{\theta}_{k}^{a}\right)_{1 \leq k \leq N}$ are equal to $\left(\widetilde{\theta}_{k}(\cdot-a)\right)_{1 \leq k \leq N}$ and the projector functions $\left(\widetilde{q}_{k}^{a}\right)_{1 \leq k \leq N}$ denote the shifted projector functions $\left(\widetilde{q}_{k}(\cdot-a)\right)_{1 \leq k \leq N}$.

Since $\widetilde{\theta}_{k}$ is an eigenfunction of $-\frac{\mathrm{d}^{2}}{\mathrm{~d} x^{2}}-Z_{0} \sum_{k \in \mathbb{Z}} \delta_{k}$, for all $1 \leq i, j \leq N$ and $I \in\{0, a\}$,

$$
\left\langle\widetilde{\theta}_{i}^{I}, H \widetilde{\theta}_{i}^{I}\right\rangle-\left\langle\widetilde{\theta}_{i}^{I}, H_{\mathrm{ps}} \widetilde{\theta}_{i}^{I}\right\rangle=-\left\langle\widetilde{\theta}_{i}^{I},-Z_{I} \chi_{\eta} \widetilde{\theta}_{i}^{I}\right\rangle .
$$

Hence, the new expression of $H_{\mathrm{ps}}^{\mathrm{PAW}}$ is given by

$$
\begin{aligned}
H_{\mathrm{ps}}^{\mathrm{PAW}}= & H_{\mathrm{ps}}+\sum_{\substack{i, j=1 \\
I \in\{0, a\}}}^{N} \widetilde{p}_{i}^{I}\left(\left\langle\phi_{i}^{I}, H \phi_{j}\right\rangle_{I, \eta}-\left\langle\widetilde{\phi}_{i}^{I}, H_{\mathrm{ps}} \widetilde{\phi}_{j}^{I}\right\rangle_{I, \eta}\right)\left\langle\widetilde{p}_{j}^{I}, \cdot\right\rangle \\
& -\sum_{\substack{i, j=1 \\
I \in\{0, a\}}}^{N} \widetilde{q}_{i}^{I}\left\langle\widetilde{\theta}_{i}^{I},-Z_{I} \chi_{\eta} \widetilde{\theta}_{j}\right\rangle_{I, \eta}\left\langle\widetilde{q}_{j}^{I}, \cdot\right\rangle,
\end{aligned}
$$

and $S^{\text {PAW }}$ remains unchanged.

We denote by $\widetilde{q}^{I}$ the vector of functions $\left(\widetilde{q}_{1}^{I}, \ldots, \widetilde{q}_{N}^{I}\right)^{T}$ and $\widetilde{\Theta}_{I}$ the vector of functions $\left(\widetilde{\theta}_{1}^{I}, \ldots, \widetilde{\theta}_{N}^{I}\right)^{T}$.

The proof of Theorem 3.5 follows the same steps of the proof of Theorem 3.4. First, we prove that for $g \in H_{\text {per }}^{1}$, the quantity $\left\langle g, H_{\mathrm{ps}}^{\mathrm{PAW}} g\right\rangle$ is equal to $\left\langle g, H^{\mathrm{VPAW}} g\right\rangle$ and error terms of the form $g-\left\langle\widetilde{p}^{I}, g\right\rangle^{T} \widetilde{\Phi}_{I}-\left\langle\widetilde{q}^{I}, g\right\rangle^{T} \widetilde{\Theta}_{I}$ that needs to be estimated.

Proposition 4.12. Let $g \in H_{\mathrm{per}}^{1}(0,1)$. Let $H_{\mathrm{ps}}^{\mathrm{PAW}}$ be the operator given by (4.31). Then

$$
\begin{aligned}
\left\langle g, H_{\mathrm{ps}}^{\mathrm{PAW}} g\right\rangle= & \left\langle g, H^{\mathrm{VPAW}} g\right\rangle-2 \sum_{I \in\{0, a\}}\left\langle g-\left\langle\widetilde{p}^{I}, g\right\rangle^{T} \widetilde{\Phi}_{I},\left\langle\widetilde{p}^{I}, g\right\rangle^{T}\left(H \Phi_{I}-(H+\delta V) \widetilde{\Phi}_{I}\right)\right\rangle \\
& +2 \sum_{I \in\{0, a\}}\left\langle g-\left\langle\widetilde{q}^{I}, g\right\rangle^{T} \widetilde{\Theta}_{I},\left\langle\widetilde{q}^{I}, g\right\rangle^{T} \delta V \widetilde{\Theta}_{I}\right\rangle_{I, \eta} \\
& +\sum_{I \in\{0, a\}}\left\langle g-\left\langle\widetilde{p}^{I}, g\right\rangle^{T} \widetilde{\Phi}_{I}-\left\langle\widetilde{q}^{I}, g\right\rangle^{T} \widetilde{\Theta}_{I}, \delta V\left(g-\left\langle\widetilde{p}^{I}, g\right\rangle^{T} \widetilde{\Phi}_{I}-\left\langle\widetilde{q}^{I}, g\right\rangle^{T} \widetilde{\Theta}_{I}\right)\right\rangle_{I, \eta} .
\end{aligned}
$$

Proof. The proof is similar to the proof of Proposition 4.5.

We now focus on the estimation of $g-\left\langle\widetilde{q}^{I}, g\right\rangle^{T} \widetilde{\Theta}_{I}$. To do so, we first need a bound on the linear form $\left\langle\widetilde{q}^{I}, g\right\rangle^{T} \widetilde{\Theta}_{I}$. 
Lemma 4.13. There exists a constant $C$ independent of $\eta$ such that for all $f \in H_{\mathrm{per}}^{1}(0,1)$ and $x \in(-\eta, \eta)$,

$$
\left|\left\langle\widetilde{q}^{I}, f\right\rangle^{T} \widetilde{\Theta}_{I}(x)\right| \leq C\|f\|_{\infty, \eta, I} .
$$

Proof. For clarity, we will drop the index $I$. For $0 \leq j \leq N-1$, let

$$
v_{j}=(2 \pi \eta)^{2 j+1}\left(\begin{array}{c}
1 \\
2^{2 j+1} \\
\vdots \\
N^{2 j+1}
\end{array}\right), \quad \hat{v}_{j}=\frac{1}{\eta^{2 j+1}} v_{j} .
$$

Let $\left(\hat{w}_{j}\right)_{0 \leq j \leq N-1}$ be the dual basis of $\left(\hat{v}_{j}\right)_{0 \leq j \leq N-1}$ and $w_{j}=\frac{1}{\eta^{2 j+1}} \hat{w}_{j}$. Let $\mathcal{M}$ be the matrix such that for all $0 \leq j, k \leq N-1$,

$$
\mathcal{M}_{j k}=\frac{(-1)^{j+k}}{(2 j+1) !(2 k+1) !} \int_{-1}^{1} \rho(t) t^{2 j+2 k+2} \mathrm{~d} t .
$$

By a Taylor expansion, we obtain for $t \in(-1,1)$,

$$
\widetilde{\Theta}(\eta t)=\left(\begin{array}{c}
\sin (2 \pi \eta t) \\
\vdots \\
\sin (2 \pi \eta N t)
\end{array}\right)=\sum_{k=0}^{N-1} \frac{(-1)^{k}(2 \pi \eta t)^{2 k+1}}{(2 k+1) !}\left(\begin{array}{c}
1 \\
2^{2 j+1} \\
\vdots \\
N^{2 j+1}
\end{array}\right)+R_{\widetilde{\Theta}}(\eta t),
$$

where $\left|R_{\widetilde{\Theta}}(\eta t)\right| \leq C \eta^{2 N+1}$. Then, we can rewrite the matrix $G$ given by (4.29) as

$$
\begin{aligned}
G & =\eta \int_{-1}^{1} \rho(t)\left(\sum_{j=0}^{N-1} \frac{(-1)^{j} \eta^{2 j+1}}{(2 j+1) !} \hat{v}_{j} t^{2 j+1}+R_{\widetilde{\Theta}}(\eta t)\right)\left(\sum_{k=0}^{N-1} \frac{(-1)^{k} \eta^{2 k+1}}{(2 k+1) !} \hat{v}_{k} t^{2 k+1}+R_{\widetilde{\Theta}}(\eta t)\right)^{T} \mathrm{~d} t \\
& =\eta \sum_{j, k=0}^{N-1} \mathcal{M}_{j k} v_{j} v_{k}^{T}+\eta \int_{-1}^{1} \sum_{j=0}^{N-1} \frac{(-1)^{j}}{(2 j+1) !} t^{2 j+1}\left(v_{j} R_{\widetilde{\Theta}}(\eta t)^{T}+R_{\widetilde{\Theta}}(\eta t) v_{j}^{T}\right) \mathrm{d} t+\mathcal{O}\left(\eta^{4 N+3}\right) .
\end{aligned}
$$

Hence, we have for $0 \leq j, k \leq N-1$,

$$
w_{j}^{T} G w_{k}=\eta \mathcal{M}_{j k}+\eta w_{j}^{T} \mathcal{R}_{k}+\eta \mathcal{R}_{j}^{T} w_{k}+\mathcal{O}\left(\eta^{5}\right),
$$

where

$$
\mathcal{R}_{k}=\int_{-1}^{1} \rho(t) \frac{(-1)^{k}}{(2 k+1) !} t^{2 k+1} R_{\widetilde{\Theta}}(\eta t) \mathrm{d} t .
$$

But $\left\|w_{k}\right\|=\mathcal{O}\left(\eta^{-2 k-1}\right)$ and $\left|R_{\widetilde{\Theta}}(\eta t)\right| \leq C \eta^{2 N+1}$, hence $\mathcal{R}_{j}^{T} w_{k}=\mathcal{O}\left(\eta^{2}\right)$. Thus, if we denote by

$$
W=\left(\begin{array}{c}
w_{0}^{T} \\
\vdots \\
w_{N-1}^{T}
\end{array}\right), \quad V=\left(\begin{array}{c}
v_{0}^{T} \\
\vdots \\
v_{N-1}^{T}
\end{array}\right),
$$

we obtain

$$
W G W^{T}=\eta \mathcal{M}+\mathcal{O}\left(\eta^{3}\right),
$$

and

$$
W^{-T} G^{-1} W^{-1}=V G^{-1} V^{T}=\frac{1}{\eta} \mathcal{M}^{-1}+\mathcal{O}(\eta)
$$


Thus, we have for $f \in L^{\infty}(-\eta, \eta)$ and $x \in(-\eta, \eta)$

$$
\begin{aligned}
\langle\widetilde{q}, f\rangle^{T} \widetilde{\Theta}(x)= & \eta\left(\int_{-1}^{1} \rho(t) f(\eta t) G^{-1}\left(\sum_{j=0}^{N-1} \frac{(-1)^{j}}{(2 j+1) !} t^{2 j+1} v_{j}+R_{\widetilde{\Theta}}(\eta t)^{T}\right) \mathrm{d} t\right)^{T} \\
& \times\left(\sum_{j=0}^{N-1} \frac{(-1)^{j}}{(2 j+1) !}\left(\frac{x}{\eta}\right)^{2 j+1} v_{j}+R_{\widetilde{\Theta}}(x)\right)
\end{aligned}
$$

By expanding (4.33), three types of terms arise involving

(1) $v_{j}^{T} G^{-1} v_{k}$ : by (4.32), we have $\left|v_{j}^{T} G^{-1} v_{k}\right|=\mathcal{O}\left(\frac{1}{\eta}\right)$;

(2) $v_{j}^{T} G^{-1} R_{\widetilde{\Theta}}(x)$ : by $(4.32),\left\|v_{j} G^{-1}\right\|=\mathcal{O}\left(\frac{1}{\eta^{2 N-1}}\right)$ and because $R_{\widetilde{\Theta}}(x)=\mathcal{O}\left(\eta^{2 N+1}\right)$, we have $\left|v_{j}^{T} G^{-1} R_{\widetilde{\Theta}}(x)\right|=$ $\mathcal{O}\left(\eta^{2}\right)$

(3) $R_{\widetilde{\Theta}}(\eta t)^{T} G^{-1} R_{\widetilde{\Theta}}(x)$ : by (4.32), we deduce that $\left\|G^{-1}\right\|=\mathcal{O}\left(\frac{1}{\eta^{4 N-1}}\right)$, but $R_{\widetilde{\Theta}}(x)=\mathcal{O}\left(\eta^{2 N+1}\right)$, hence $\left|R_{\widetilde{\Theta}}(\eta t)^{T} G^{-1} R_{\widetilde{\Theta}}(x)\right|=\mathcal{O}\left(\eta^{3}\right)$.

Hence we finally obtain

$$
\left|\langle\widetilde{q}, f\rangle^{T} \widetilde{\Theta}(x)\right| \leq C\|f\|_{\infty, \eta}
$$

Lemma 4.14. Let $f$ be a smooth and odd function. Then we have

$$
\left\|f-\left\langle\widetilde{q}^{I}, f\right\rangle^{T} \widetilde{\Theta}_{I}\right\|_{\infty, \eta, I} \leq C \eta^{2 N+3}\left\|f^{(2 N+3)}\right\|_{\infty, \eta, I} .
$$

Proof. The proof of this lemma is similar to the proof of Lemma 4.9. Hence it boils down to the estimation of $f$ by its best approximation on $[-I+\eta, I+\eta], I \in\{0, a\}$ of $f$ by a linear combination of the odd PAW functions $\theta_{k}$. We can prove that this best approximation is determined by cancelling the first orders appearing in the Taylor expansion around $I$ of $f$. As we can cancel all the orders up to $2 N+1$, the difference between the best approximation and the function $f$ in the $L^{\infty}$-norm is of order $\mathcal{O}\left(\eta^{2 N+3}\right)$. We conclude the proof of this lemma using Lemma 4.13.

The presence of $\widetilde{\theta}_{j}$ and $\widetilde{q}_{j}$ (see (4.31) above) does not change the lower bound of the PAW eigenvalue as it does not improve the estimate of critical terms in the proof of the lower bound in Theorem 3.4. From the proof of the upper bound of Theorem 3.4, it appears that only the odd part of the VPAW generalized eigenfunction $\widetilde{\psi}$ prevents to have a better bound. This is due to the absence of odd PAW functions in the PAW method. With these odd PAW functions, using Lemma 4.14, denoting by $\widetilde{\psi}_{o}$ the odd part of the VPAW eigenfunction, we have $\left\|\widetilde{\psi}_{o}-\left\langle\widetilde{q}^{I}, \widetilde{\psi}_{o}\right\rangle^{T} \widetilde{\Theta}_{I}\right\|_{\infty, \eta, I} \leq C \eta^{2 N+3}\left\|\widetilde{\psi}_{o}^{(2 N+3)}\right\|_{\infty, \eta, I}$. Since $\phi_{i}^{I}$ and $\widetilde{\phi}_{i}^{I}$ are even functions, $\widetilde{\psi}_{o}$ is equal to the odd part of the original eigenfunction $\psi$. Hence $\left\|\widetilde{\psi}_{o}^{(2 N+3)}\right\|_{\infty, \eta, I}$ can be bounded independently of $\eta$. Using this approximation result on the odd part of the VPAW eigenfunction with Proposition 4.12, we have the upper bound of Theorem 3.5 .

\section{NUMERICAL TESTS}

In this section, some numerical tests are provided to confirm the theoretical bounds obtained in Theorems 3.33.5. The simulations of the different PAW versions are done with $a=0.4$ and $Z_{0}=Z_{a}=10$. The regularity of the pseudo wave functions $\widetilde{\phi}_{i}^{I}$ is set to $d=6$. 


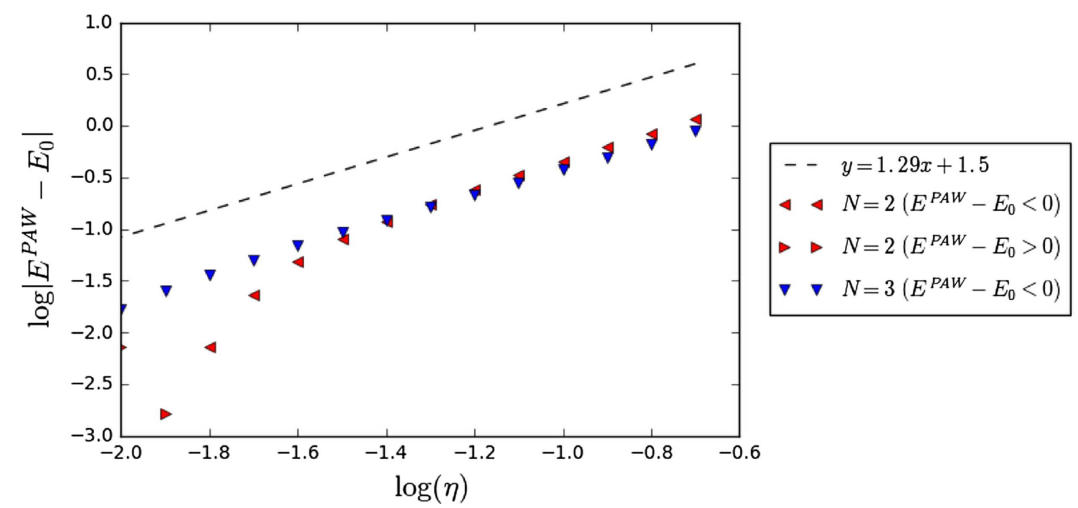

Figure 1. Error on the lowest eigenvalue of the truncated PAW equations (2.9).

\subsection{The PAW equations}

\subsubsection{Without pseudopotentials}

We solve the generalized eigenvalue problem

$$
H^{\mathrm{PAW}} f=E^{\mathrm{PAW}} S^{\mathrm{PAW}} f
$$

where $H^{\text {PAW }}$ and $S^{\text {PAW }}$ are defined by equations (2.10) and (2.11), by expanding $f$ in 512 plane-waves. We study how $E^{\mathrm{PAW}}$ behaves as a function of $\eta$. In our case, the PAW eigenvalue $E^{\mathrm{PAW}}$ can be smaller than $E_{0}$, which shows that the PAW method is not variational. When $E^{\text {PAW }}-E_{0}<0$, Theorem 3.3 states that $E^{\text {PAW }}$ converges at least linearly to $E_{0}$. This is what we observe in Figure 1.

\subsubsection{With pseudopotentials}

The eigenfunction $f$ is expanded in 1000 plane waves for which convergence is reached. The Fourier coefficients of the PAW functions are computed using an accurate numerical integral scheme. The function $\chi$ used for the pseudopotential $\chi_{\epsilon}$ is

$$
\forall x \in[-1,1], \quad \chi(x)=\frac{\mathrm{e}^{1 /\left(1-x^{2}\right)}}{\int_{-1}^{1} \mathrm{e}^{1 /\left(1-y^{2}\right)} \mathrm{d} y} .
$$

The parameter $\epsilon$ of the size of the support of the pseudopotential is equal to the PAW radius $\eta$.

In view of Figure 2, the lower bound in Theorem 3.4 seems sharp. The use of odd PAW functions improves the error on the PAW eigenvalue (Fig. 3) for a range of moderate values of the cut-off radius $\eta$. However, the use of odd PAW functions does not give a better lower bound.

Finally, the upper bound in Theorem 3.5 seems optimal (see Fig. 3). For $N=2$, we have a slope close to the theoretical value $(2 N=4)$.

\subsection{Comparison between the PAW and VPAW methods in pre-asymptotic regime}

Using Fourier methods to solve the VPAW eigenvalue problem (2.15), we have the following bound on the computed eigenvalue $E_{M}^{\mathrm{VPAW}}[3]$ :

$$
0<E_{M}^{\mathrm{VPAW}}-E_{0} \leq C\left(\frac{\eta^{4 N}}{M}+\frac{1}{\eta^{2 d-2}} \frac{1}{M^{2 d-1}}\right)
$$

where $M$ is the number of plane-waves, $N$ the number of PAW functions and $d$ the regularity of the PAW pseudo wave functions $\widetilde{\phi}_{k}$. 


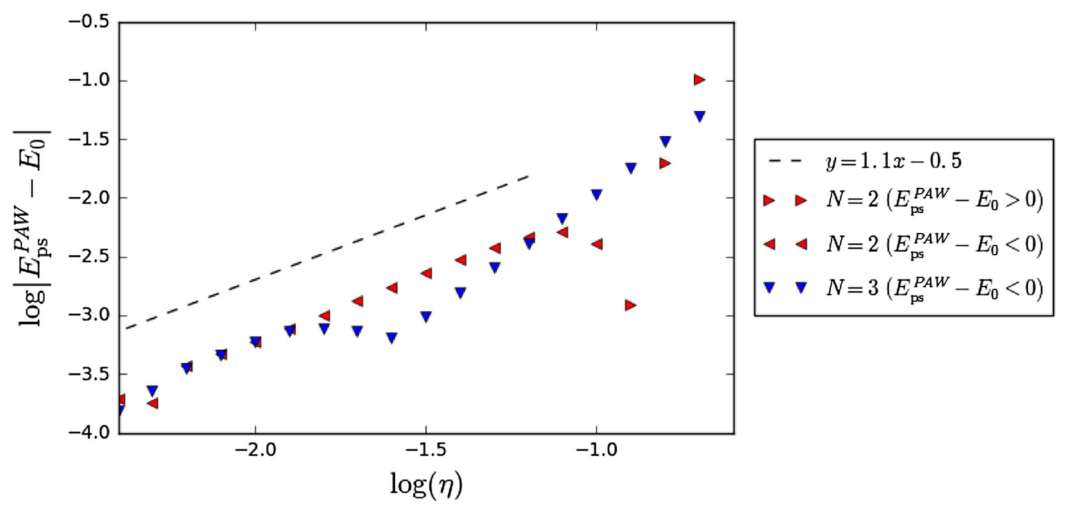

Figure 2. Error on the lowest eigenvalue of the PAW equations (2.12) with pseudopotentials.

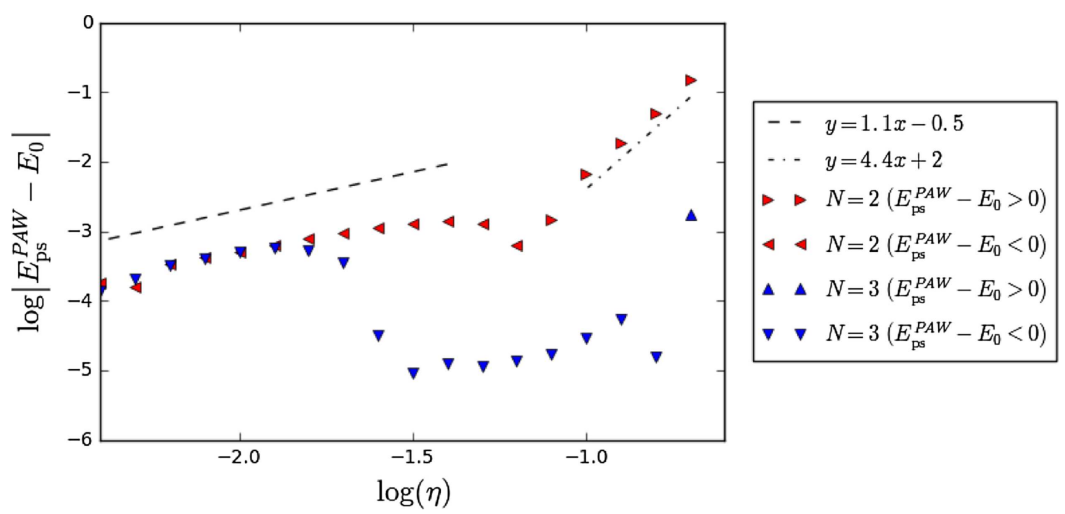

FiguRE 3. Error on the lowest eigenvalue of the PAW equations with pseudopotentials including odd PAW functions.

In Figure $4, E_{0}$ is the lowest eigenvalue of the 1D-Schrödinger operator $H$. The PAW method considered in Figure 4 is the generalized eigenvalue problem (2.12). The simulations are run for a fixed value of $d=6$ and different values of $\eta=0.1$ and $\eta=0.2$.

As expected, the PAW method quickly stagnates to $E_{\mathrm{ps}}^{\mathrm{PAW}}$ which, according to Theorem 3.4, is close but not equal to $E_{0}$. Although the VPAW method does not remove the Dirac singularities -which is why, asymptotically, the VPAW method convergence rate is of order $\mathcal{O}\left(\frac{1}{M}\right)$, it converges faster to $E_{0}$ than the PAW method with pseudopotentials. The plane-wave convergence of the VPAW method is sensitive to the choice of the cut-off radius $\eta$. For small values of the cut-off radius, the plane-wave convergence is fast and of order $\mathcal{O}\left(\frac{1}{M^{2 d-1}}\right)$. The prefactor is however larger since it scales as $\frac{1}{\eta^{2 d-2}}$. For small plane-wave cut-off, it can hence be preferable to use a larger cut-off radius $\eta$ for a better accuracy.

\section{Conclusion}

In this paper, error estimates of the PAW method applied to a one-dimensional toy model reproducing the difficulties due to the cusps has been proved. These estimates are supported by numerical evidences.

A similar analysis of the PAW method applied to periodic Hamiltonians with Coulomb potentials should be achievable. The fundamental relation between the PAW and the VPAW Hamiltonians (Props. 4.4 and 4.5) holds 

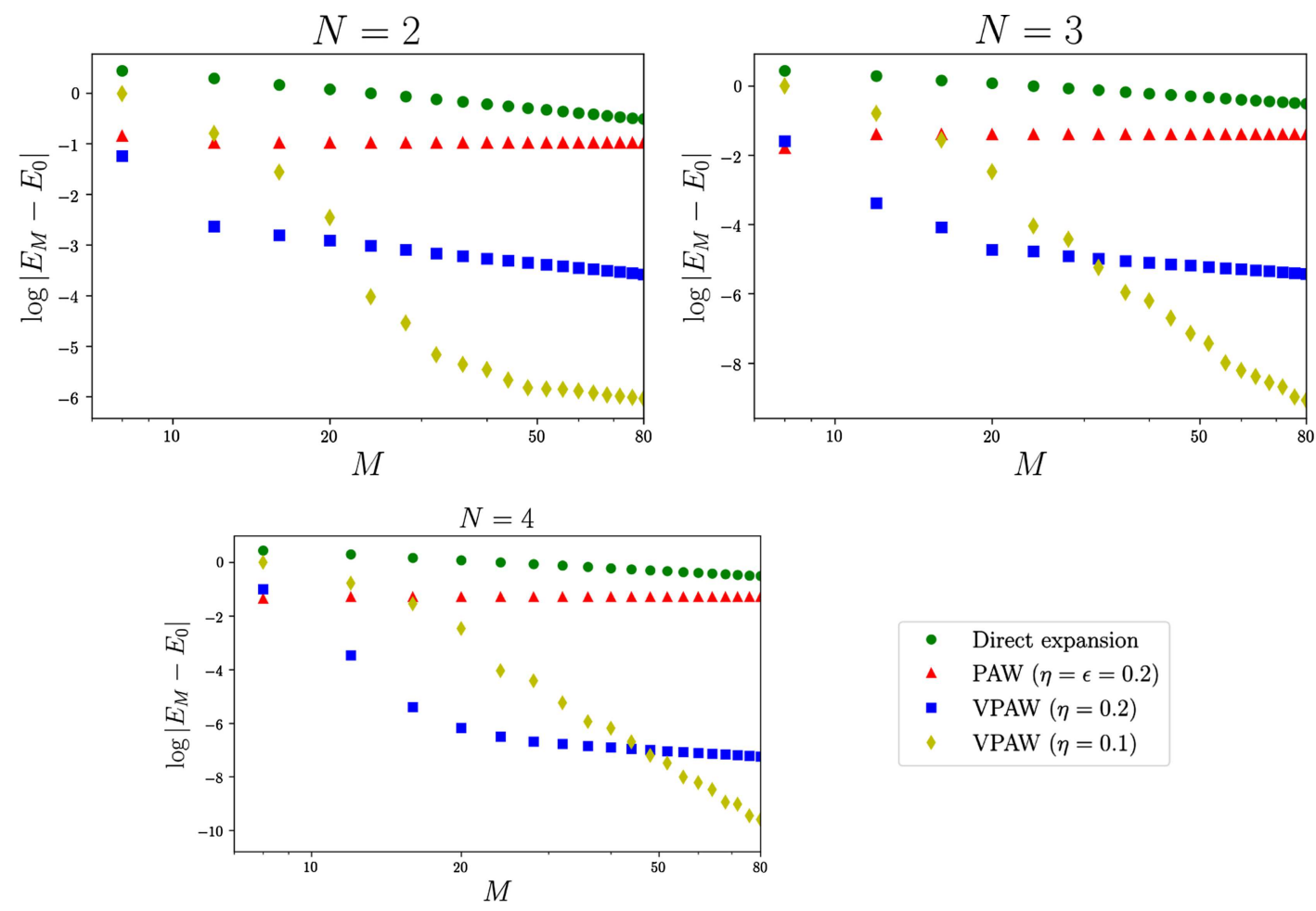

- Direct expansion

\ $\operatorname{PAW}(\eta=\epsilon=0.2)$

- $\operatorname{VPAW}(\eta=0.2)$

- $\operatorname{VPAW}(\eta=0.1)$

Figure 4. Comparison between the PAW and VPAW methods.

for more general setting. A study of the VPAW method for three-dimensional Hamiltonian is in preparation, which is crucial for the analysis of the PAW method.

\section{Appendix A. Bounds on expressions involving PAW functions}

We have gathered in this section some technical results involving PAW functions. Most of these results can be found in [3] or are corollaries of lemmas in this paper.

Lemma A.1. Let $\widetilde{\psi}$ be an eigenfunction of (2.1) associated to the lowest eigenvalue $E_{0}$ and $\widetilde{\psi}_{e}$ be its even part. Let $\psi=\left(\operatorname{Id}+T_{N}\right) \widetilde{\psi}$ where $T_{N}$ is the operator (2.16) and $\psi_{e}$ be the even part of $\psi$. Under Assumptions 3.1 and 3.2, there exists a constant $C$ independent of $\eta$ such that for any $0<\eta \leq \eta_{0}$ we have

$$
\left\|\widetilde{\psi}_{e}-\left\langle\widetilde{p}^{I}, \widetilde{\psi}\right\rangle^{T} \widetilde{\Phi}_{I}\right\|_{\infty, \eta, I} \leq C \eta^{2 N}
$$

and

$$
\left\|E_{0} \psi_{e}-\left(\left\langle A_{I}^{-1} p^{I}, \psi\right\rangle\right)^{T} \mathcal{E}^{I} \Phi_{I}\right\|_{\infty, \eta, I} \leq C \eta^{2 N-2},
$$

where $\mathcal{E}^{I}$ is the $N \times N$ diagonal matrix with entries $\left(-\epsilon_{1}^{I}, \ldots,-\epsilon_{N}^{I}\right)$.

Proof. In the neighbourhood of $I, I \in\{0, a\}$ by definition of the operator $T_{I, N}$ in (2.17), we have

$$
\widetilde{\psi}-\left\langle\widetilde{p}^{I}, \widetilde{\psi}\right\rangle^{T} \widetilde{\Phi}_{I}=\psi-\left\langle\widetilde{p}^{I}, \widetilde{\psi}\right\rangle^{T} \Phi_{I}
$$


Multiplying this equation by $\widetilde{p}_{i}^{I}$ for some $1 \leq i \leq N$, integrating over $[I-\eta, I+\eta]$ and using the duality between the pseudo wave functions $\widetilde{\phi}_{j}^{I}$ and the projector functions $\widetilde{p}_{j}^{I}$, we obtain

$$
\left\langle\widetilde{p}_{i}^{I}, \psi\right\rangle=\sum_{j=1}^{N}\left\langle\widetilde{p}_{i}^{I}, \phi_{j}^{I}\right\rangle\left\langle\widetilde{p}_{j}^{I}, \widetilde{\psi}\right\rangle .
$$

Let $\widetilde{A}_{I}=\left(\left\langle\widetilde{p}_{i}^{I}, \phi_{j}^{I}\right\rangle\right)_{1 \leq i, j \leq N}$. Writing the last equation in a matrix-vector format, we have

$$
\left\langle\widetilde{p}^{I}, \widetilde{\psi}\right\rangle=\widetilde{A}_{I}^{-1}\left\langle\widetilde{p}^{I}, \psi\right\rangle
$$

By definition of the projector functions, $\widetilde{A}_{I}^{-1}\left\langle\widetilde{p}^{I}, \psi\right\rangle=A_{I}^{-1}\left\langle p^{I}, \psi\right\rangle$. Hence we have

$$
\widetilde{\psi}-\left\langle\widetilde{p}^{I}, \widetilde{\psi}\right\rangle^{T} \widetilde{\Phi}_{I}=\psi-\left\langle A_{I}^{-1} p^{I}, \psi\right\rangle^{T} \Phi_{I},
$$

and in combination with Lemmas 4.2 and 4.6 in [3], we obtain

$$
\left\|\widetilde{\psi}_{e}-\left\langle\widetilde{p}^{I}, \widetilde{\psi}\right\rangle^{T} \widetilde{\Phi}_{I}\right\|_{\infty, \eta, I} \leq C \eta^{2 N}
$$

where $C>0$ is independent of $\eta$.

The second estimate is proved the same way.

Lemma A.2. Let $P_{k}(t)=\frac{1}{2^{k} k !}\left(t^{2}-1\right)^{k}$ and $P(t)=\left(P_{0}(t), \ldots, P_{d-1}(t)\right)^{T}$. Let $C_{\eta}^{(P)} \in \mathbb{R}^{N \times d}, d \geq N$ be the matrix such that for $t \in(-\eta, \eta)$,

$$
\widetilde{\Phi}_{I}(t)=C_{\eta}^{(P)} P\left(\frac{t}{\eta}\right)
$$

Let $C_{1} \in \mathbb{R}^{N \times N}$ and $C_{2} \in \mathbb{R}^{N \times(d-N)}$ be the matrices such that

$$
C_{\eta}^{(P)}=\left(C_{1} \mid C_{2}\right)
$$

Let $M_{\eta} \in \mathbb{R}^{d \times d}$ be the matrix

$$
M_{\eta}=\left(C_{\eta}^{(P)}\right)^{T}\left(C_{\eta}^{(P)} G(P)\left(C_{\eta}^{(P)}\right)^{T}\right)^{-1} C_{\eta}^{(P)},
$$

where $G(P)$ is the matrix $\int_{-1}^{1} \rho(t) P(t) P(t)^{T} \mathrm{~d} t$.

Then the following statements hold.

(1) the norm of the matrix $M_{\eta}$ is uniformly bounded in $\eta$.

(2) for all $x \in(-\eta, \eta)$

$$
\left\langle\widetilde{p}^{I}, f\right\rangle^{T} \Phi_{I}(x)=\left(M_{\eta} \int_{-1}^{1} \rho(t) f(\eta t) P(t) \mathrm{d} t\right)^{T}\left(\begin{array}{c}
C_{1}^{-1} \\
0
\end{array}\right) \Phi_{I}(x),
$$

and

$$
\left\langle\widetilde{p}^{I}, f\right\rangle^{T} \widetilde{\Phi}_{I}(x)=\left(M_{\eta} \int_{-1}^{1} \rho(t) f(\eta t) P(t) \mathrm{d} t\right)^{T} P(x / \eta)
$$


(3) for all $0<\eta \leq \eta_{0}$ and $x \in(-\eta, \eta)$

$$
C_{1}^{-1} \Phi_{I}(x)=\left(\begin{array}{l}
1 \\
*
\end{array}\right)+\mathcal{O}(\eta) \text { and } C_{1}^{-1} \Phi_{I}^{\prime}(x)=\frac{1}{\eta}\left(\begin{array}{l}
0 \\
*
\end{array}\right)+\mathcal{O}(1)
$$

where $\left(\begin{array}{l}1 \\ *\end{array}\right)$ and $\left(\begin{array}{l}0 \\ *\end{array}\right)$ are vectors of $\mathbb{R}^{N}$ with a first entry equal to 1 (resp. 0) and are uniformly bounded in $\eta$ and $x$.

Proof. Proofs of these statements can be found in the proof of Lemmas 4.13 and 4.14 in [3].

Lemma A.3. There exists a positive constant $C$ independent of $f$ and $\eta$ such that we have the following estimates

(1) for all $f \in H_{\mathrm{per}}^{1}(0,1), 0<\eta \leq \eta_{0}$ and $x \in\left(-\frac{1}{2}, \frac{1}{2}\right)$, we have

$$
\left|\left\langle\widetilde{p}^{I}, f\right\rangle^{T}\left(\Phi_{I}(x)-\widetilde{\Phi}_{I}(x)\right)\right| \leq C \eta\|f\|_{H_{\mathrm{per}}^{1}} \quad \text { and } \quad\left|\left\langle\widetilde{p}^{I}, f\right\rangle^{T}\left(\Phi_{I}^{\prime}(x)-\widetilde{\Phi}_{I}^{\prime}(x)\right)\right| \leq C\|f\|_{H_{\mathrm{per}}^{1}} ;
$$

(2) for all $f \in L_{\mathrm{per}}^{2}(0,1), 0<\eta \leq \eta_{0}$ and $x \in\left(-\frac{1}{2}, \frac{1}{2}\right)$, we have

$$
\left|\left\langle\widetilde{p}^{I}, f\right\rangle^{T}\left(\Phi_{I}(x)-\widetilde{\Phi}_{I}(x)\right)\right| \leq \frac{C}{\eta^{1 / 2}}\|f\|_{L_{\mathrm{per}}^{2}} ;
$$

(3) for all $f \in H_{\mathrm{per}}^{1}(0,1), 0<\eta \leq \eta_{0}$ and $x \in(-\eta, \eta)$, we have

$$
\left|\left\langle\widetilde{p}^{I}, f\right\rangle^{T} \widetilde{\Phi}_{I}(x)\right| \leq C\|f\|_{L^{\infty}} \quad \text { and } \quad\left|\left\langle\widetilde{p}^{I}, f\right\rangle^{T} \Phi_{I}(x)\right| \leq C\|f\|_{L^{\infty}} ;
$$

(4) for all $f \in H_{\mathrm{per}}^{1}(0,1), 0<\eta \leq \eta_{0}$ and $x \in(-\eta, \eta)$, we have

$$
\left|\left\langle\widetilde{p}^{I}, f\right\rangle^{T} \widetilde{\Phi}_{I}^{\prime}(x)\right| \leq C\|f\|_{H_{\mathrm{per}}^{1}} \quad \text { and } \quad\left|\left\langle\widetilde{p}^{I}, f\right\rangle^{T} \Phi_{I}^{\prime}(x)\right| \leq C\|f\|_{H_{\mathrm{per}}^{1}} .
$$

Proof. (1) Proof of this statement can be found in [3] (Lems. 4.12 and 4.14).

(2) By Lemma A.2,

$$
\left\langle\widetilde{p}^{I}, f\right\rangle^{T}\left(\Phi_{I}(x)-\widetilde{\Phi}_{I}(x)\right)=\left(M_{\eta} \int_{-1}^{1} \rho(t) f(\eta t) P(t) \mathrm{d} t\right)^{T}\left(\left(\begin{array}{c}
C_{1}^{-1} \\
0
\end{array}\right) \Phi_{I}(x)-P(x / \eta)\right) .
$$

Applying the Cauchy-Schwarz inequality to $\int_{-1}^{1} \rho(t) f(\eta t) P(t) \mathrm{d} t$ suffices to prove the estimate.

(3) By item 2 of Lemma A.2,

$$
\left\langle\widetilde{p}^{I}, f\right\rangle^{T} \widetilde{\Phi}_{I}(x)=\left(M_{\eta} \int_{-1}^{1} \rho(t) f(\eta t) P(t) \mathrm{d} t\right)^{T} P(x / \eta) .
$$

Thus the first inequality follows from the uniform boundedness of $M_{\eta}$ with respect to $\eta$ (item 1 of Lemma A.2). For the second inequality, we proceed the same way and conclude using item 3 of Lemma A.2.

(4) For the first inequality, we simply replace Step 1 in the proof of Lemma 4.12 in [3] by

1. $\frac{1}{\eta} P^{\prime}(x / \eta)=\frac{1}{\eta}\left(\begin{array}{l}0 \\ *\end{array}\right)+\mathcal{O}(1)$

and keep on the proof. For the second inequality, we replace Step 1 in the proof of Lemma 4.12 in [3] by item 3 of Lemma A.2. 


\section{Appendix B. Results on the operators $S^{\mathrm{VPAW}}$ And $S^{\mathrm{PAW}}$}

Some properties of the operators $S^{\mathrm{VPAW}}$ and $S^{\mathrm{PAW}}$ are stated in the next lemma.

Lemma B.1. The operators $S^{\mathrm{VPAW}}$ and $S^{\mathrm{PAW}}$ satisfies the following properties. There exists a constant $C$ independent of $\eta$ such that for all $f \in H_{\mathrm{per}}^{1}(0,1)$ :

$$
\begin{gathered}
\left|\left\langle f, S^{\mathrm{VPAW}} f\right\rangle\right| \leq C\|f\|_{L_{\mathrm{per}}^{2}}^{2} . \\
\left|\left\langle f, S^{\mathrm{PAW}} f\right\rangle\right| \leq C\|f\|_{L_{\mathrm{per}}^{2}}^{2} . \\
\left|\left\langle f, S^{\mathrm{VPAW}} f\right\rangle-\left\langle f, S^{\mathrm{PAW}} f\right\rangle\right| \leq C \eta^{2}\|f\|_{H_{\mathrm{per}}^{1}}^{2} .
\end{gathered}
$$

(4) let $\widetilde{\psi}$ be a generalized eigenfunction of $(2.15)$

$$
\left|\left\langle\widetilde{\psi}, S^{\mathrm{VPAW}} \widetilde{\psi}\right\rangle-\left\langle\widetilde{\psi}, S^{\mathrm{PAW}} \widetilde{\psi}\right\rangle\right| \leq C \eta^{2 N+2}\|\widetilde{\psi}\|_{H_{\mathrm{per}}^{1}}
$$

$$
\left|\left\langle f, S^{\mathrm{PAW}} f\right\rangle-\langle f, f\rangle\right| \leq C \eta\|f\|_{H_{\mathrm{per}}^{1}}^{2} .
$$

Proof. (1) By item 2 of Lemma A.3, there exists a constant $C$ independent of $\eta$ and $x$ such that for all $x \in\left(-\frac{1}{2}, \frac{1}{2}\right)$ and for all $0<\eta \leq \eta_{0}$

$$
\left|\left\langle\widetilde{p}^{I}, f\right\rangle^{T}\left(\Phi_{I}(x)-\widetilde{\Phi}_{I}(x)\right)\right| \leq \frac{C}{\eta^{1 / 2}}\|f\|_{L_{\mathrm{per}}^{2}} .
$$

Then from (2.17), we have

$$
\begin{aligned}
\left\|T_{0, N} f\right\|_{L_{\mathrm{per}}^{2}}^{2} & =\int_{0}^{1}\left|\left\langle\widetilde{p}^{0}, f\right\rangle^{T}\left(\Phi^{0}(x)-\widetilde{\Phi}^{0}(x)\right)\right|^{2} \mathrm{~d} x \\
& \leq \int_{-\eta}^{\eta}\left|\left\langle\widetilde{p}^{0}, f\right\rangle^{T}\left(\Phi^{0}(x)-\widetilde{\Phi}^{0}(x)\right)\right|^{2} \mathrm{~d} x \\
& \leq C\|f\|_{L_{\text {per }}^{2}}^{2} .
\end{aligned}
$$

Similarly, $\left\|T_{a, N} f\right\|_{L_{\mathrm{per}}^{2}} \leq C\|f\|_{L_{\mathrm{per}}^{2}}$. Thus

$$
\begin{aligned}
\left\langle S^{\mathrm{VPAW}} f, f\right\rangle & =\left\langle\left(\mathrm{Id}+T_{N}\right) f,\left(\mathrm{Id}+T_{N}\right) f\right\rangle=\left\|\left(\mathrm{Id}+T_{N}\right) f\right\|_{L_{\mathrm{per}}^{2}}^{2}=\left\|\left(\mathrm{Id}+T_{0, N}+T_{a, N}\right) f\right\|_{L_{\mathrm{per}}^{2}}^{2} \\
& \leq C\|f\|_{L_{\mathrm{per}}^{2}}^{2} .
\end{aligned}
$$

(2) By Proposition 4.1, for all $f \in H_{\mathrm{per}}^{1}(0,1)$

$$
\left\langle f, S^{\mathrm{PAW}} f\right\rangle=\left\langle f, S^{\mathrm{VPAW}} f\right\rangle-2 \sum_{I=\{0, a\}}\left\langle\left\langle\widetilde{p}^{I}, f\right\rangle^{T}\left(\Phi_{I}-\widetilde{\Phi}_{I}\right), f-\left\langle\widetilde{p}^{I}, f\right\rangle^{T} \widetilde{\Phi}_{I}\right\rangle .
$$

From items 1 and 2 of Lemma A.2, it is easy to show that there exists a constant $C$ independent of $\eta$ and $x$ such that for all $x \in(-\eta, \eta), 0<\eta \leq \eta_{0}$ and $f \in H_{\text {per }}^{1}(0,1)$

$$
\left|\left\langle\widetilde{p}^{I}, f\right\rangle^{T} \widetilde{\Phi}_{I}(x)\right| \leq \frac{C}{\eta^{1 / 2}}\|f\|_{L_{\mathrm{per}}^{2}} .
$$


Hence

$$
\begin{aligned}
\left|\left\langle f, S^{\mathrm{PAW}} f\right\rangle\right| & \leq\left|\left\langle f, S^{\mathrm{VPAW}} f\right\rangle\right|+2 \sum_{I \in\{0, a\}}\left|\left\langle\left\langle\widetilde{p}^{I}, f\right\rangle^{T}\left(\Phi_{I}-\widetilde{\Phi}_{I}\right), f-\left\langle\widetilde{p}^{I}, f\right\rangle^{T} \widetilde{\Phi}_{I}\right\rangle\right| \\
& \leq C\|f\|_{L_{\mathrm{per}}^{2}}^{2}+\sum_{I \in\{0, a\}}\left\|\left\langle\widetilde{p}^{I}, f\right\rangle^{T}\left(\Phi_{I}-\widetilde{\Phi}_{I}\right)\right\|_{L_{\mathrm{per}}^{2}}\left(\|f\|_{L_{\mathrm{per}}^{2}}+\left\|\left\langle\widetilde{p}^{I}, f\right\rangle^{T} \widetilde{\Phi}_{I}\right\|_{2, \eta, I}\right) \\
& \leq C\|f\|_{L_{\mathrm{per}}^{2}}^{2} .
\end{aligned}
$$

(3) This is a consequence of Proposition 4.1 and items 1 and 3 of Lemma A.3.

(4) By Proposition 4.1

$$
\left\langle\widetilde{\psi}, S^{\mathrm{PAW}} \widetilde{\psi}\right\rangle=\left\langle\widetilde{\psi}, S^{\mathrm{VPAW}} \widetilde{\psi}\right\rangle-2 \sum_{I \in\{0, a\}}\left\langle\left\langle\widetilde{p}^{I}, \widetilde{\psi}\right\rangle^{T}\left(\Phi_{I}-\widetilde{\Phi}_{I}\right), \widetilde{\psi}-\left\langle\widetilde{p}^{I}, \widetilde{\psi}\right\rangle^{T} \widetilde{\Phi}_{I}\right\rangle .
$$

By Lemma A.1, we have for each $I \in\{0, a\}$

$$
\left\|\widetilde{\psi}_{e}-\left\langle\widetilde{p}^{I}, \widetilde{\psi}\right\rangle^{T} \widetilde{\Phi}_{I}\right\|_{\infty, \eta, I} \leq C \eta^{2 N}
$$

where $\widetilde{\psi}_{e}$ denotes the even part of $\widetilde{\psi}$ and $C>0$ is independent of $\eta$. Hence, using the parity of the PAW functions $\phi_{k}$ and $\widetilde{\phi}_{k}$ and item 1 of Lemma A.3,

$$
\begin{aligned}
\left|\left\langle\left\langle\widetilde{p}^{I}, \widetilde{\psi}\right\rangle^{T}\left(\Phi_{I}-\widetilde{\Phi}_{I}\right), \widetilde{\psi}-\left\langle\widetilde{p}^{I}, \widetilde{\psi}\right\rangle^{T} \widetilde{\Phi}_{I}\right\rangle\right| & =\left|\left\langle\left\langle\widetilde{p}^{I}, \widetilde{\psi}\right\rangle^{T}\left(\Phi_{I}-\widetilde{\Phi}_{I}\right), \widetilde{\psi}_{e}-\left\langle\widetilde{p}^{I}, \widetilde{\psi}\right\rangle^{T} \widetilde{\Phi}_{I}\right\rangle\right| \\
& \leq\left\|\left\langle\widetilde{p}^{I}, \widetilde{\psi}\right\rangle^{T}\left(\Phi_{I}-\widetilde{\Phi}_{I}\right)\right\|_{1, \eta, I} \| \widetilde{\psi}-\left\langle\widetilde{p}^{I}, \widetilde{\psi}^{T} \widetilde{\Phi}_{I} \|_{\infty, \eta, I}\right. \\
& \leq C \eta^{2 N+2}\|\widetilde{\psi}\|_{H_{\mathrm{per}}^{1}} .
\end{aligned}
$$

and the result follows.

(5) By item 3 of Lemma B.1, we have for all $f \in H_{\text {per }}^{1}(0,1)$

$$
\left|\left\langle f, S^{\mathrm{VPAW}} f\right\rangle-\left\langle f, S^{\mathrm{PAW}} f\right\rangle\right| \leq C \eta^{2}\|f\|_{H_{\mathrm{per}}^{1}}^{2},
$$

where $C$ is a constant independent of $\eta$ and $f$.

Using the definition of the operator $S^{\mathrm{VPAW}}$ in (2.19) and item 1 of Lemma A.3, we can show that

$$
\left|\left\langle f, S^{\mathrm{VPAW}} f\right\rangle-\langle f, f\rangle\right| \leq C \eta\|f\|_{H_{\mathrm{per}}^{1}}^{2},
$$

with a constant $C$ independent of $\eta$ and $f$. By a triangular inequality, the result follows.

Acknowledgements. The author is grateful to Xavier Blanc and Eric Cancés for interesting and helpful discussions. The author would also like to thank the anonymous referees for their comments which significantly improved the clarity of the paper.

\section{REFERENCES}

[1] C. Audouze, L'utilisation du formalisme PAW en théorie de la fonctionnelle de la densité perturbée. Research Report CEA (2006).

[2] C. Audouze, F. Jollet, M. Torrent and X. Gonze, Projector augmented-wave approach to density-functional perturbation theory. Phys. Rev. B $\mathbf{7 3}$ (2006) 235101. 
[3] X. Blanc, E. Cancès and M.-S. Dupuy, Variational projector augmented-wave method: theoretical analysis and preliminary numerical results. Preprint arXiv:1711.06529 (2017).

[4] X. Blanc, E. Cancès and M.-S. Dupuy, Variational projector augmented-wave method. C.R. Math. 355 (2017) 665-670.

[5] P.E. Blochl, Projector augmented-wave method. Phys. Rev. B 50 (1994) 17953-17979.

[6] E. Cancès and G. Dusson, Discretization error cancellation in electronic structure calculation: toward a quantitative study. ESAIM: M2AN 51 (2017) 1617-1636.

[7] N. Holzwarth, A. Tackett and G. Matthews, A Projector Augmented Wave (PAW) code for electronic structure calculations, part I: atompaw for generating atom-centered functions. Comput. Phys. Commun. 135 (2001) 329-347.

[8] F. Jollet, M. Torrent and N. Holzwarth, Generation of projector augmented-wave atomic data: A 71 element validated table in the XML format. Comput. Phys. Commun. 185 (2014) 1246-1254.

[9] T. Kato, On the eigenfunctions of many-particle systems in quantum mechanics. Commun. Pure Appl. Math. 10 (1957) $151-177$.

[10] L. Kleinman and D. Bylander, Efficacious form for model pseudopotentials. Phys. Rev. Lett. 48 (1982) 1425.

[11] G. Kresse and D. Joubert, From ultrasoft pseudopotentials to the projector augmented-wave method. Phys. Rev. B 59 (1999) $1758-1775$.

[12] C.J. Pickard and F. Mauri, All-electron magnetic response with pseudopotentials: NMR chemical shifts. Phys. Rev. B 63 (2001) 245101.

[13] M. Torrent, F. Jollet, F. Bottin, G. Zérah and X. Gonze, Implementation of the projector augmented-wave method in the ABINIT code: application to the study of iron under pressure. Comput. Mater. Sci. 42 (2008) 337-351.

[14] N. Troullier and J.L. Martins, Efficient pseudopotentials for plane-wave calculations. Phys. Rev. B 43 (1991) 1993. 